\title{
Genome-wide identification and expression analysis of ADP- ribosylation factors associated with biotic and abiotic stress in wheat (Triticum aestivum L.)
}

\author{
Yaqian Li ${ }^{1,2}$, Jinghan Song ${ }^{1,2}$, Guang Zhu ${ }^{1}$, Zehao Hou ${ }^{2}$, Lin Wang ${ }^{2}$, Xiaoxue Wu ${ }^{2}$, Zhengwu Fang $^{2}$, Yike Liu ${ }^{\text {Corresp., }}$ \\ 1,2, Chunbao Gao ${ }^{1,2}$ \\ ${ }^{1}$ Hubei Key Laboratory of Food Crop Germplasm and Genetic Improvement, Food Crops Institute, Hubei Academy of Agricultural Sciences/Hubei \\ Engineering and Technology Research Center of Wheat/Wheat Disease Biology Research Station for Central China, Wuhan, China \\ 2 Engineering Research Center of Ecology and Agricultural Use of Wetland, Ministry of Education/Hubei Collaborative Innovation Center for Grain \\ Industry/College of Agriculture, Yangtze University, Jingzhou, China \\ Corresponding Author: Yike Liu \\ Email address: hbliuyk@foxmail.com
}

The ARF gene family plays important roles in intracellular transport in eukaryotes and is involved in conferring tolerance to biotic and abiotic stresses in plants. To explore the role of these genes in the development of wheat (Triticum aestivum L.), 74 wheat ARF genes (TaARFs; including 18 alternate transcripts) were identified and clustered into 7 subgroups. Phylogenetic analysis revealed that TaARFAl sub-group genes were strongly conserved. Numerous cis-elements functionally associated with the stress response and hormones were identified in the TaARFAl sub-group, implying that these TaARFs are induced in response to abiotic and biotic stresses in wheat. According to available transcriptome data and qRT-PCR analysis, the TaARFAl genes displayed tissue-specific expression patterns and were regulated by biotic stress (powdery mildew and stripe rust) and abiotic stress (cold, heat, $\mathrm{ABA}$, drought and $\mathrm{NaCl}$ ). Protein interaction network analysis further indicated that TaARFAl proteins may interact with protein phosphatase 2C (PP2C), which is a key protein in the ABA signaling pathway. This comprehensive analysis will be useful for further functional characterization of TaARF genes and the development of highquality wheat varieties. 


\section{Genome-wide identification and expression analysis of ADP-}

\section{2 ribosylation factors associated with biotic and abiotic stress in}

\section{3 wheat (Triticum aestivum L.)}

4 Yaqian $\mathrm{Li}^{1,2}$, Jinghan Song ${ }^{1,2}$, Guang $\mathrm{Zhu}^{1}{ }^{1}$,Zehao $\mathrm{Hou}^{2}$, Lin Wang ${ }^{2}$, Xiaoxue $\mathrm{Wu}^{2}$, Zhengwu Fang ${ }^{2}$, Yike

5 Liu ${ }^{1,2, *}$, Chunbao Gao ${ }^{1,2}$

1 Hubei Key Laboratory of Food Crop Germplasm and Genetic Improvement, Food Crops Institute, Hubei Academy of Agricultural Sciences/Hubei Engineering and Technology Research Center of Wheat/Wheat Disease Biology Research Station for Central China, Wuhan, China; 201972386@yangtzeu.edu.cn (Y. L); songjingh@126.com(J. S); guangnianzhu@163.com (G. Z); gcbgybwj@163.com(C. G)

2 Engineering Research Center of Ecology and Agricultural Use of Wetland, Ministry of Education/Hubei Collaborative Innovation Center for Grain Industry/College of Agriculture, Yangtze University, Jingzhou 434000, Hubei, China; 201771374@yangtzeu.edu.cn (Z. H); wl2310484554@163.com (L.W); 1315148616@qq.com(X.W); fangzhengwu88@163.com (Z.F)

Corresponding Author: Yike Liu

3 Nanhu Avenue, Wuhan, Hubei, 430064, China

Email address: hbliuyk@foxmail.com 


\section{Abstract}

7 The $A R F$ gene family plays important roles in intracellular transport in eukaryotes and is

8 involved in conferring tolerance to biotic and abiotic stresses in plants. To explore the role of 9 these genes in the development of wheat (Triticum aestivum L.), 74 wheat ARF genes (TaARFs; including 18 alternate transcripts) were identified and clustered into 7 sub-groups. Phylogenetic analysis revealed that TaARFA1 sub-group genes were strongly conserved. Numerous ciselements functionally associated with the stress response and hormones were identified in the TaARFA1 sub-group, implying that these TaARFs are induced in response to abiotic and biotic stresses in wheat. According to available transcriptome data and qRT-PCR analysis, the TaARFA1 genes displayed tissue-specific expression patterns and were regulated by biotic stress (powdery mildew and stripe rust) and abiotic stress (cold, heat, $\mathrm{ABA}$, drought and $\mathrm{NaCl}$ ). Protein interaction network analysis further indicated that TaARFA1 proteins may interact with protein phosphatase 2C (PP2C), which is a key protein in the ABA signaling pathway. This comprehensive analysis will be useful for further functional characterization of $T a A R F$ genes and the development of high-quality wheat varieties.

Keywords: Wheat; TaARF; Genome-wide; Phylogenetic analysis; Gene expression

\section{Introduction}

Intracellular transport is crucial for cell survival and growth, and promotes the formation of cell membranes and lysosomes to enable the secretion of proteins, hormones, and neurotransmitters, and absorb exogenous molecules by endocytosis. The transmembrane transport of macromolecules and granular substances in eukaryotic cells is carried out by encapsulating them in vesicles with a lipid bilayer. Vesicle transport is carefully controlled by regulatory molecules, including ADP-ribosylation factors (ARFs) (Boman et al., 2000). ARFs are allosteric activators of cholera toxin, and are in an activated state when bound to GTP, and in a deactivated state when bound to GDP (Wonderlich et al., 2011; Bourgoin, Azreq 2012). ARFs belong to the Ras superfamily, which is divided into five subfamilies: Ras, Rho, Rab, Ran, and ARF (Burgoyne, 2001). The $A R F$ subfamily lacks the $\mathrm{C}$-terminal isoprenylation and carboxymethylation regions, which is different from other members of the Ras superfamily, but possesses an additional nucleotide-sensitive region, an extended N-terminus, and a covalently attached myristate, which complement each other to constitute a 'myristoyl switch' (Bérauddufour et al., 1999). GTP hydrolysis dissociates ARF, which then coats proteins on the membrane so that the vesicles can dock and fuse with target membranes (Gebbie et al., 2005). Their molecular mass is approximately $21 \mathrm{kDa}$, and the $A R F$ gene family were classified into $A R F s$ and $A R F$-like (ARL) genes based on their amino acid (aa) sequence homology (Bourgoin, Azreq, 2012; Muthamilarasan et al., 2016). ARF proteins are highly conserved ( $>60 \%$ similarity among themselves) and have similar biological activities, while ARL proteins are highly divergent (40$60 \%$ identity) and play roles in different pathways, including secretory pathways ( $L i$ et al., 2004). 
46 The primary structure of cholera toxin is highly conserved, and the toxin plays important roles in 47 intracellular transport in eukaryotes (Mi Hee et al., 2002). For example, in Arabidopsis, the 48 absence of $A R F 1$ in the Golgi apparatus results in the development of an abnormal structure, 49 inhibition of protein transport, and inhibition of plant growth and development (Myung Ki et al., 50 2013). ARFs also play roles in resisting biotic and abiotic stresses in plants (Muthamilarasan et 51 al., 2016; Lee et al., 2003). ARFs in rice may be related to stress resistance, and ARF1 might 52 participate in various plant defense signaling pathways (Lee et al., 2003). Overexpression of 53 ARF1 confers salt and drought tolerance in rice, Arabadopsis transgenic plants, and Spartina 54 alterniflora. (Joshi et al., 2014).

55

56

Common wheat (Triticum aestivum L.) is one of the most important crops for humans, and occupies a large production area, providing staple food globally (Veraverbeke, Delcour, 2002; Shiferaw et al., 2013). However, the quality of wheat is often affected by adverse environmental conditions, including drought, salt stress, heavy metals, low temperature, pests, and diseases (Bajwa et al., 2018). Considering the important roles of ARFs in plant growth and development, and response to abiotic and biotic stresses, a comprehensive understanding of $A R F S$ in wheat ( $T a A R F S$ ) would contribute to functional understanding of $A R F S$ and support resistance breeding. In the present study, Chinese Spring genome data (IWGSC RefSeq v1.1) was used to conduct a systematic and comprehensive phylogenetic analysis of the TaARFs, including the gene structures, conserved motifs, chromosomal locations, cis-elements, synteny, and duplication patterns of ARF protein sequences. The STRING database was used to generate protein-protein networks between TaARF proteins so as to better understand gene functions. Furthermore, based on the available transcriptome data and qRT-PCR analysis, we analyzed the expression profiles of TaARFs at different development stages and under different abiotic and biotic stresses to predict their potential functions and regulatory patterns, which provides a theory evidence for wheat stress resistance gene breeding.

74

75

76

77

78

79

80

81

82

83

84

85

\section{Materials \& Methods}

\section{Identification of ARF genes}

Computer-based methods were used to identify members of the $A R F$ gene family from the wheat reference genome IWGSC RefSeq v1.1 assembly (https://wheat-urgi.versailles.inra.fr). Known ARF protein sequences, including 19 ARFs from Arabidopsis thaliana (AtARFs) and 21 ARFs from Oryza sativa (OsARFs), were used as query sequences for BLASTp analysis with an evalue cutoff of $<1 \times 10^{-10}$ (Altschul et al., 1997). The obtained sequences were submitted to InterProScan (http://www.ebi.ac.uk/interpro/) to check the ARF domains (Mulder, Apweiler, 2007). The Pfam database (http://pfam.xfam.org/) was used to select sequences that contained the ARF-box domain (PF00025) (Finn et al., 2006). The hit sequences were further validated using SMART (http://smart.embl-heidelberg.de/smart) to remove unmatched proteins (Letunic et al., 2004). 
86 Phylogenetic analysis and characterization of TaARFs

87 A total of 96 protein sequences (19 from Arabidopsis (Gebbie et al., 2005), 21 from rice 88 (Muthamilarasan et al., 2016), and 56 from wheat) were compared by ClustalW2 software with 89 default parameters (Oliver et al., 2005). An unrooted phylogenetic tree was created using the 90 Neighbor-Joining (NJ) method with 1000 replicated-bootstraps in MEGA 7.0 software (Kumar, 91 Stecher \& Tamura, 2016). Then, the phylogenetic tree was edited by the Interactive Tree of Life 92 (ITOL, Version 3.2.317, http://itol.embl.de/) to have the final illustration (Letunic, Bork, 2016). 93 The identified TaARFs were used to perform protein characterization in ExPASy Server10 94 (http://prosite. expasy.org/) (Wilkins et al., 1999). The predicted protein features for each of the 95 96 protein sequences were determined, including the length, molecular weight (MW), instability index, isoelectric point (pI), and aliphatic index. Plant-mPLoc (http://www.csbio.sjtu.edu.cn/bioinf/plant-multi/) was used to predict the cellular localization (Horton et al., 2006).

105

106

107

108

109

110

111

112

113

114

115

116

117

118

119

120

121

122

123

124

125

\section{Exon-intron structure and motif analysis of $\mathrm{TaARFS}$}

Structural analysis was performed to identify the exon-intron structure of each TaARF gene using GSDS (http://gsds.cbi.pku.edu.cn/index.php/) (Hu et al., 2015). The software MEME (http://meme-suite.org/) was used to determine conserved TaARF motifs (Bailey et al., 2006). Motifs with E-values $>0.001$ were probably statistical artefacts rather than real motifs, and were excluded (Bailey et al., 2006). The parameters were employed as the following descriptions: the maximum number of motifs, 10; and the optimum width of each motif, between 6 and 50 residues. The motif prediction results were inserted into TBtools (https://github.com/CJChen/TBtools/) to produce the illustrations (Chen et al., 2018). The locations of conserved domains were determined by SMART and visualized in MEME to reveal the diversification of TaARFs (Fang et al., 2020).

\section{Chromosomal locations and synteny of TaARFs}

The location information of TaARF genes were obtained from the reference genome in the IWGSC v1.1 database. Moreover, we generated chromosome locations using MapInspect version 1.0. The common tool "all against all BLAST search" was used to determine possible paralogous or orthologous sequences among wheat sequences with an E-value cutoff of 1e-10 and identity $>80 \%$ (Gu et al., 2002). The R package "circlize" was employed to prepare a diagram showing the locations and homology relationships of TaARFs (Zuguang et al., 2014). The non-synonymous $(\mathrm{Ka})$ and synonymous $(\mathrm{Ks})$ substitution rates were calculated using DNA Sequence Polymorphism (DnaSP) 5.10 to analyze gene duplication events (Rozas, 2009). We calculated the $\mathrm{Ka} / \mathrm{Ks}$ ratios for the replicated $A R F$ gene pairs in wheat to explore whether Darwinian positive selection pressure has affected the functional proportion of replicated genes. We also compared the gene duplication events of $A R F$ genes between wheat and Triticum dicoccoides, Aegilops tauschii, Arabidopsis, and rice. In general, when the ratio is greater than 1, the replicated gene is under positive selection, a ratio equal to 1 indicates genes under neutral 
126 evolution, and a ratio of less than 1 indicates genes under negative selection pressure (Zhang et

127

128

129

130

131

132

133

134

135

136

137

138

139

140

141

142

143

144

145

146

147

148

149

150

151

152

153

154

155

156

157

158

159

160

161

162

163

164

165

al., 2006; Anton, Kateryna \& Li, 2002).

\section{Multiple conditional transcriptome analysis of $\mathrm{TaARFS}$}

Multiple RNA-seq data from different tissues, development stages, and treatments were downloaded from the NCBI Short Read Archive (SRA) database and mapped to the wheat genome using HISAT2. Cufflinks were used to perform gene assembly, expression level calculations, and identifications of differences in differentially expressed genes (Cole et al., 2012). The obtained transcripts per million (TPM) values reflecting the expression level of each gene were used to generate a heatmap of TaARFs using the R package "pheatmap" (Kolde, 2015). Triad expression analysis was carried out as described previously (Ramírez-González et al., 2018).

\section{Plant materials and qRT-PCR}

Hexaploid wheat (cultivar Emai 170; Triticum aestivum; AABBDD) was used to validate the expression patterns of selected candidate genes in all experiments. Seeds were surface sterilized with $1 \%$ hydrogen peroxide, germinated in an incubator at $28^{\circ} \mathrm{C}$ for $2 \mathrm{~d}$, and transferred to a greenhouse at $26^{\circ} \mathrm{C}$ with a $16 \mathrm{~h}$ light $/ 8 \mathrm{~h}$ dark cycle and relative humidity of $60-70 \%$. Seven-dayold seedlings were treated with cold, $\mathrm{ABA}$, and $\mathrm{NaCl}$. Control seedlings continued to grow under standard conditions. Root tissue was collected $6 \mathrm{~h}$ and $12 \mathrm{~h}$ after treatment, including the set controls. All sample materials were quickly frozen in liquid nitrogen and stored at $-80^{\circ} \mathrm{C}$ before RNA extraction. Total RNA was isolated using the Trizol reagent (Invitrogen, Carlsbad, CA, USA). Quantitative real time (RT)-PCR was performed using the TaKaRa qRT-PCR system (RR047A). The gene-specific primers (Table S10) were designed using Primer 5.0 to amplify 80-350 bp fragments. The thermal cycling conditions were as follows: $95^{\circ} \mathrm{C}$ for $30 \mathrm{~s}$ and 40 cycles of $95^{\circ} \mathrm{C}$ for $5 \mathrm{~s}$ and $60^{\circ} \mathrm{C}$ for $30 \mathrm{~s}$. The relative quantity of target gene transcripts was calculated using the $2^{-} \triangle \triangle \mathrm{ct}$ method with wheat $\beta$-actin and GAPDH as reference genes (Yin et al., 2018).

\section{Cis-element analysis of putative promoter regions}

The cis-elements in the promoter regions are related to gene expression patterns and functions (Anne-Laure, Adrien \& Veitia, 2014). To investigate the cis-elements in the promoter regions of genes of interest, we downloaded $1.5 \mathrm{~kb}$ of the genomic DNA sequences upstream of the start codon corresponding to each gene from the hexaploid wheat database. The Plant CARE database (http://bioinformatics.psb.ugent.be/webtools/plantcare/html/) were used to analysis the putative cis-elements in the promoter sequences (Magali et al., 2002).

\section{Gene Ontology annotation and protein interaction network}

A GO (Gene Ontology) database was used for functional annotation of the ARF genes using MAJORBIO CLOUD (https://cloud.majorbio.com/). GO annotations were mapped according to biological processes, molecular functions, and cellular components. TaARFs protein-protein 
166

167

168

169

170

171

172

173

174

175

176

177

178

179

180

181

182

183

184

185

186

187

188

189

190

191

192

193

194

195

196

197

198

199

200

201

202

203

204

205

interaction networks was assembled using the STRING tool (https://string-db.org/cgi). All predicted TaARF proteins have been submitted to the STRING database. The minimum required interaction score was set to high confidence $(0.900)$. The active interaction sources was setted come from 'experiments' and 'databases'. The maximum number of interactors were no more than 5 on the first shell.

\section{Results}

\section{Identification and classification of ARF genes in wheat}

In the present study, the entire hexaploid wheat genome was downloaded and used to construct a local database. After genomic retrieval, a total of 126 proteins that were similar to ARF were obtained from hexaploid wheat; however, only 77 wheat sequences were confirmed to be conserved in the ARF family domain (PF00025 and IPR006689) by Pfam and InterProScan. Sequences without complete conserved domains or with a length less than 170 aa were excluded. After elimination, 74 ARF proteins containing complete domains were obtained. Among the remaining ARF proteins, 18 were splice variants (Table S1). To investigate their evolutionary relationships, we constructed an NJ tree with MEGA7.0 using the amino acid sequences of putative $A R F$ family members from Arabidopsis, rice, and wheat (Fig. 1, Table S2). The predicted TaARF proteins were classified into seven sub-groups: ARFA1, ARFB1, ARFC1, ARF3, ARL1, TTN5, and GB1 based on the phylogenetic tree and previous reports (Muthamilarasan et al., 2016). The ARL1 sub-group is the largest of these sub-groups with 17 members. The ARFA1 and the ARFB1 sub-groups have 14 and 9 members, respectively. There are 3 proteins in the ARF3 and ARFC1 sub-groups, and the other sub-groups each contain 5 members. Interestingly, Arabidopsis had one ARFD1 protein, while rice and wheat did not have any. These results are consistent with the coevolutionary relationships between these species, indicating that the relationship between wheat and rice is closer than that with Arabidopsis. Each TaARF gene was named based on its phylogenetic relationship with AtARFs and OsARFs (Muthamilarasan et al., 2016). Genes corresponded equally across the three homoeologous subgenomes (A, B, and D) in wheat, which is referred to as a triad (Ramírez-González et al., 2018). We identified 15 TaARFs triads with reference to the results of Ramírez-González et al (Ramírez-González et al., 2018) (Table S3). TaARFs triads have identical gene names except for the sub-genome identifier (A, B or D).

\section{Features of predicted TaARF proteins}

Of the 56 putative TaARF proteins, the predicted MW was around $21 \mathrm{kDa}$ on average, except for TaARFA1a-D and TaARFB1c-B. The number of exons and introns were similar within the same class but different between classes. The protein lengths ranged from 170 (TaGB1b-B) to 263 (TaARFA1a-D) amino acids (aa) and the predicted isoelectric point ranged from 5.67 to 9.35 (Table 1). The protein instability index shows that $82 \%$ TaARFs are stable proteins, but TaARFA1a-D, TaARFB1b-A, TaARFB1c-B, TaARFB1d-B, TaARFB1e-N, TaGB1a-A, TaGB1a-B, TaGB1a-D, TaGB1b-B, and TaGB1c-D were predicted to be unstable. The average 
206 hydropathicity (GRAVY) was less than 0 , except in the case of TaARFC1-A/B/D, indicating that 207 most of these proteins are hydrophilic (Table S1). Subcellular localization prediction showed that 208 TaARFs are localized mainly in the cytoplasm, but are also found in the chloroplasts, 209 peroxisome, nucleus, or mitochondria (Table S1).

210

211 Gene structure and motif analysis of the TaARF gene family

212 The structure of genes can be used to predict their expression and function. We found that 213 TaARFs contained different exon-intron composition patterns by comparing the gene structures 214 (Fig. 2). There were $38 T a A R F$ sequences with both untranslated regions (UTRs), and of the 215 remaining sequences, 14 did not have 5'- and 3'-UTRs, 2 (TaARFAld-A and TaARFA1c-D) had 216 only 5'-UTRs, and 2 (TaARFAla-D and TaARFB1b-A) had only 3'-UTRs (Fig. 2B). The number 217 of introns ranged from 1 to 7 , and $43 \%$ contained 6 exons of varying lengths. TaARF genes in the 218 same sub-group shared similar exon-intron structure. The majority of the ARFB1 sub-group 219 contained 6 exons, with the exception TaARFBId-B, which contains five. While the ARFA1 220 subgroup gene members have 5 exons, ARFB1, ARFC1, and ARF3 subgroups have 6, 2 and 8 221 exons, respectively.

222 The 10 most statistically significant motifs were chosen to describe the motif pattern in TaARFs, 223 which were named motif 1-10 (Fig. 2C, Table S4). The lengths of the 10 motifs were between 8 224 (motif 8 and 9) and 49 (motif 1) aa residues. The number of motifs in each TaARF protein varied 225 from 5 to 8 . Notably, motif analysis indicated that most TaARFs have relatively conserved motif 226 compositions. All TaARF proteins (except TaGB1c-D and TaARFB1d-B) contained motif 1 to 4, 227 which contained the ARF-box domain. In general, many TaARFs of the same group encoded 228

229

230

231

232

233

234

235

236

237

238

239

240

241

242

243

244

245 proteins with similar motif compositions, and therefore, these proteins may have similar functions. There were 4 motifs (motif 1, 3, 4, and 5) in the middle region of most TaARF proteins; however, different motifs were found at the N-terminal and C-terminal regions. For example, two specific motifs (motif 9 and motif 10) were only found in the C-terminus of the TaARL1 sub-group. Motif 7 appeared in both the N-terminus and the C-terminus of the TaARL1 subfamily, but only in the C-terminus of other subfamilies.

\section{Chromosomal distribution and gene duplication events of TaARF genes}

To further investigate the genetic differences in the TaARF gene family, we mapped their chromosomal locations. After positioning, 55 TaARFs were mapped on all 21 chromosomes, while TaARFBIe- $N$ was not mapped on any chromosomes (Fig. 3A, Table 1). TaARFs were distributed roughly evenly across the three subgenomes (subgenome A, 18; subgenome B, 18; subgenome D, 19). Members from the same sub-group tended to be distributed at similar locations. However, the distribution of genes on chromosomes varied from one homoeologous group to another. The largest homoeologous group of 5 chromosomes (A, B, and D) had the most TaARF genes (12), followed by the smallest homoeologous groups of 2 (4 genes) and 7 (5 genes). By screening the sequence identity and position, 75 segmental duplication pairs were predicted, with no tandem duplication pairs being identified. Triads were not considered when 
246 predicting gene duplication events. The $\mathrm{Ka} / \mathrm{Ks}$ ratio between $T a A R F$ gene pairs was less than 1 247 (average 0.08) in all cases (Fig. 3B, Table S5), suggesting that the TaARF gene family might 248 have experienced strong purifying selective pressure (Song et al., 2019). Among the TaARF 249 genes, 75 pairs of segmental duplication genes were found concentrated in the ARFA1 subgroup 250 (Table S5).

251 Four comparative syntenic maps of wheat associated with four representative species, including 252 Triticum dicoccoides, Arabidopsis, Aegilops tauschii, and rice, were constructed to further 253 deduce the evolutionary origin and orthologous relationship of the wheat ARF family (Fig. 4). 254 The numbers of orthologous pairs between the other four species (Triticum dicoccoides, Aegilops 255 256 tauschii, Arabidopsis, and rice) were 61, 27, 67, and 88, respectively (Table S6). Six TaARF genes have both orthologous genes in four species. Among them, the ARFA1 subgroup accounted for 5 genes, which was a larger number than the other subfamilies.

\section{Expression analysis of TaARF genes in different tissues}

RNA-sequencing (RNA-seq) is a powerful tool to explore transcription patterns using highthroughput sequencing (Wang, Gerstein \& Snyder, 2009). The RNA-seq data for five tissues (grain, spike, leaf, stem, and root) in wheat were used to characterize the expression of TaARF genes during growth and development. Out of the 56 full-length genes, $93 \%$ were expressed in at least one developmental stage, with a wide expression range between 1-852 TPM (TPM $\left.\mathrm{max}_{\max }\right)($ Fig. $5 \mathrm{~A}$; Table S1, S7). The remaining 7\% of full-length genes had a very low level of expression with a $\mathrm{TPM}_{\max }<1$, and were considered as "not expressed" (Fig. S1, Table S1). In general, the expression of TaARFs could be divided into three patterns: the first group contains members that are widely expressed in many tissues during multiple developmental stages, the second group contains those that are highly induced only at specific growth and development stages, and the last group contained members with no expression or low expression during all growth and developmental phases. Almost all $A R F$ genes in the sub-group ARFA1 were highly expressed in multiple tissues, and may therefore be involved in the regulation of growth and development.

The differential abundance of homoeologs was analyzed using a previously described framework (Ramírez-González et al., 2018). Balanced expression of homoeolog triads was denoted when transcripts from every gene had a similar abundance. Suppressed and dominant categories were denoted if some transcripts were more abundant than others (Fig. 5B, S2). Expression data were obtained from a developmental time course of Chinese Spring wheat (Ramírez-González et al., 2018). The percentage of triads in the balanced category was between $60 \%$ and $73 \%$, with an average of $68.3 \%$ (Table S8), which is consist with the values observed for transcripts from all wheat genes (Ramirez-González et al., 2018). BAR software was used to display the electron fluorescence diagram of TaARFAla-A expression to better understand TaARF gene expression during growth and development. Our results suggest that some TaARFs may play an important role during wheat growth (Fig. 5C).

84 Expression analysis of TaARF genes under biotic and abiotic stresses 
285

286

287

288

289

290

291

292

293

294

295

296

297

298

299

300

301

302

303

304

305

306

307

308

309

310

311

312

313

314

315

316

317

318

319

320

321

322

323

324

The TaARFA1 sub-group members that had higher expression during different developmental stages were selected for further analysis. The original RNA-seq data related to abiotic stress (drought and heat) and biotic stress (powdery mildew and stripe rust) from the NCBI database was used for expression profiling of TaARFA1 sub-group members (Table S9). The levels of TaARFAl genes were up-regulated after $6 \mathrm{~h}$ of drought treatments compared with the control, and then down-regulated with prolonged exposure to stress (Fig. 6A). The pattern of expression was opposite under high temperature conditions, with down-regulation followed by up-regulation to near-control levels after $12 \mathrm{~h}$. With drought and heat co-treatment, TaARFAl genes showed similar expression patterns to under high temperature alone, although the magnitude of downregulation was greater with multiple stressors. During biological stress, the expression levels of most TaARFA1 sub-group genes fluctuated. Powdery mildew infection caused the expression of TaARFAl genes (except TaARFAle-A/B/D) to be up-regulated compared to the control. The expression level of TaARFAle-A/B/D were down-regulated after infection for $24 \mathrm{~h}$, then upregulated with prolonged treatment, exceeding that of the control after $72 \mathrm{~h}$ (Fig. 6B). The magnitude of changes in expression during stripe rust infection were much smaller than during powdery mildew infection.

Due to the lack of transcriptome data for $\mathrm{ABA}$ and osmotic stress in the roots, we selected TaARFA1 genes for qRT-PCR analysis under cold, salt, and ABA stress (Table S10, 11). The qRT-PCR results revealed that TaARFAl genes were responsive to all abiotic stress treatments, and their expression patterns varied based on the stress type. Under ABA treatment, the expression levels of most TaARFAl genes were down-regulated compared with the control, except the expression levels of TaARFAlb-A, TaARFAlc-D, and TaARFAle-A/B/D, which increased after $6 \mathrm{~h}$ of treatment but recovered to the control level after $12 \mathrm{~h}$. The expression levels of TaARFA1c-D, TaARFAld-A/B/D, and TaARFAle-A/B were significantly up-regulated during $\mathrm{NaCl}$ and cold treatment, while TaARFAlf-A/B/D were down-regulated (Fig. 7).

\section{Cis-acting elements in the promoter of TaARF}

The distribution of different cis-acting elements in gene promoters may reflect differences in function and regulation. Identified cis-acting elements were divided into three major categories, namely those relating to growth and development, phytohormone response, and biotic/abiotic stress (Table S12,13). Among them, the CAAT-box and TATA-box were the most frequently observed, and related to growth and development. We predicted 3 cis-acting elements related to biotic/abiotic stress (ARE, G-box, and Sp1) and phytohormone response (ABRE, CGTCA motif, and TGACG motif) in TaARFA1 genes (Fig. 8A). The cis-acting elements related to growth and development were enriched in TaARFAl genes (Fig. 8B).

\section{Gene ontology annotation and protein interaction network of TaARF genes}

Gene ontology (GO) annotation is currently one of the most important functional annotation methods. In the present dataset, GO terms related to: (1) biological processes (BP), including regulation of biological process (GO: 0050789), response to stimulus (GO: 0050896), single- 
325

326

327

328

329

330

331

332

333

334

335

336

337

338

339

340

341

342

343

344

345

346

347

348

349

350

351

352

353

354

355

356

357

358

359

360

361

362

363

364

organism process (GO: 0044699), cellular process (GO: 0009987), signaling (GO: 0023052), biological regulation (GO: 0065007); (2) cellular components (CC), including cell (GO:0005623), organelle (GO:0043226), cell part (GO:0044464); and (3) molecular function (MF), including binding(GO:0005488), were specifically enriched (Fig. 9A, Table S14). In this study, we also used string website to predict the protein interaction network in which the TaARF genes were involved (Fig. S3). Proteins that share similar functions or participate in the same pathway tend to show interaction networks, so gene clusters or modules were formed in the network of protein interactions. It's obviously that TaARFA1 and PP2C proteins may have interaction relationship (Fig. 9B). PP2C is a key protein in the ABA signaling pathway; in the absence of ABA, PP2Cs were negatively regulated by repressors that suppress gene transcription (Nguyen, Jung \& Cheong, 2019). TaARFA1 genes might interact with PP2C to respond to biotic and abiotic stress.

\section{Discussion}

Many plant genomes have been analyzed during the continuous maturation of sequencing technology, allowing the identification of gene families at the whole genome level (Takayuki A et al., 2005; Wang et al., 2018). ARFs function in diverse physiological and molecular activities, and recent evidence has demonstrated their involvement in conferring tolerance to biotic and abiotic stresses in plants (Muthamilarasan et al., 2016). A genome-wide analysis of the TaARF gene family was performed previously, and 56, 30, 13, 19, and 21 ARF members were identified in hexaploid wheat, Triticum dicoccoides, Aegilops tauschii, Arabidopsis thaliana, and rice, respectively (Muthamilarasan et al., 2016). The number of TaARF genes is higher than other plants, possibly because wheat is a heterologous hexaploid, which has experienced two expansions during its evolution. Most genes belonging to triads in TaARF genes were located at similar positions on homoeologous chromosomes, supporting the theory that polyploidization events may have played a role in the expansion of TaARF numbers (Zengcui et al., 2011). In the process of polyploidization, a large number of $T a A R F$ genes may be created by gene replication and the conservation of $T a A R F$ genes. Multiple copies of $A R F$ genes may have been retained in wheat because they endow plants with abiotic and biological resistance.

Duplication is also an important evolutionary process in gene family expansion, and duplicated genetic material provides opportunities for functional differentiation (Jiang et al., 2019; Santoshkumar et al., 2013). During evolution, duplicated gene pairs can experience functional divergence, contributing to the formation of new gene functions, which is essential for environmental adaptability and speciation (Conant, Wolfe 2008; Victoria E, F Bryan 2002). The analysis of gene duplication could help us to understand the evolution of genes and species. According to the Holub, a chromosomal region containing two or more copies of the same gene within $200 \mathrm{~kb}$ is defined as a tandem duplication event, or a segmental duplication event (Holub, 2001; Jessica et al., 2007). In the present study, all gene duplications were segmental duplication and most segmentally duplicated gene pairs were from the same group, and located at similar 
365

366

367

368

369

370

371

372

373

374

375

376

377

378

379

380

381

382

383

384

385

386

387

388

389

390

391

392

393

394

395

396

397

398

399

400

401

402

403

404

positions in homoeologous chromosomes. In addition, duplicated genes were concentrated in the ARFA1 sub-group. The $\mathrm{Ka} / \mathrm{Ks}$ ratios of wheat ARF genes were less than 1 (average 0.08), implying that all duplicated gene pairs were under negative selection pressure. Many of the TaARFs in segmentally duplicated pairs exhibited similar exon structures. We also constructed four comparative syntenic maps of wheat associated with four representative species to further deduce the evolutionary origin and orthologous relationships of the TaARF gene family. Among the orthologous genes, six TaARF genes have both orthologous genes in four species. Among them, the ARFA1 subgroup accounts for 5 genes, suggesting that $A R F A 1$ subgroup genes may play a crucial role. These results also show that these genes originate from an ancestral gene, and may have been generated by gene replication, which is more evolutionarily conservative and therefore more commonly observed in genes that are essential for survival. In plants possessing multiple copies of $A R F$ genes, the protein content or mRNA expression would be consequently increased, possibly resulting in increased resistance to stress. Some studies have shown that increased gene dosage is beneficial to plant resistance, for example, plants with higher copy numbers of glyphosate resistance genes have stronger resistance to glyphosate (Widholm et al., 2001). In general, the TaARF gene family was constrained by evolution to maintain its functional stability.

RNA-seq analysis provides insights into the expression patterns of genes in different development stages and under a variety of stress conditions (Ramirez-González et al., 2018). In the present study, we analyzed TaARFAl expression in different plant organs and determined that this subgroup had relatively high transcript accumulation, which supports their direct or indirect involvement in certain developmental stages. TaARFAIb-A and TaARFBIa-A/B/D were relatively highly expressed in root tissue. Interestingly, there are $4 T a A R F$ genes that expressed at very low levels, indicating that they may not play a role in wheat growth and development. The ARF gene family can also respond to biotic and abiotic stresses in plants (Muthamilarasan et al., 2016). Plants are subjected to many stresses, causing survival pressure that can reduce wheat yield and negatively impact social-economic stability (Chen et al., 2015; Nussbaumer et al., 2015). Analysis of RNA-seq data revealed that most TaARFs had a similar expression profile and were either significantly up- or down-regulated under the tested stress conditions, supporting the fact that some of these environmental stresses share similar regulatory responses and signal transduction pathways (Ma, Bohnert 2007). These genes may potentially play shared roles in stress resistance.

We selected TaARFAl genes in the root and performed qRT-PCR to validate the expression pattern that we had determined using publicly available RNA-seq data. Perhaps unsurprisingly, we found that different TaARF genes responded to different stresses with different expression patterns, similarly to the RNA-seq data. The gene expression patterns under different abiotic stresses were distinct, implying that the signaling pathways involved in these responses are stress-specific (Zhao et al., 2018). The expression levels of TaARFAld-A/D in the root decreased

Peer) reviewing PDF | (2020:10:54615:1:1:NEW 30 Dec 2020) 
405

406

407

408

409

410

411

412

413

414

415

416

417

418

419

420

421

422

423

424

425

426

427

428

429

430

431

432

433

434

435

436

437

438

439

440

441

442

443

444

with $\mathrm{ABA}$ treatment, but increased with $\mathrm{NaCl}$ and cold treatment. This specific stress response warrants further investigation to identify the underlying molecular mechanisms.

By predicting the interaction network of wheat ARF protein, we found that all ARFA1 members interact with PP2C, which participates in plant growth and development and also plays major roles in the response to biotic and abiotic stresses, including bacterial pathogens (Ivy et al., 2010), salt (Yuzuki Manabe et al., 2007), drought (Kazuo Shinozaki, Yamaguchi-Shinozaki 2006), and abscisic acid (ABA) (K Meyer, MP Leube \& Grill, 1994). TaPP2C-a10 transgenic Arabidopsis exhibited decreased tolerance to drought stress (Yu et al., 2020). Overexpression of maize ZmPP2C in Arabidopsis decreased ABA sensitivity and plant drought tolerance (Liu et al., 2009). PP2Cs are abscisic acid (ABA) co-receptors that negatively regulate the ABA signaling pathway by inhibiting downstream SnRK2 protein kinases (Sreenivasuluab et al., 2012). ARFA1 may therefore suppress the expression of $P P 2 C$ genes.

\section{Conclusions}

In the present study, a total of 56 TaARFs (excluding 18 splice variants) were identified with relatively conserved motifs within sub-groups. The $\mathrm{Ka} / \mathrm{Ks}$ ratio of all gene pairs was less than 1 , indicating that $T a A R F$ genes are under negative selection pressure. Gene duplication events were concentrated on the ARFA1 sub-group, suggesting that TaARFAl genes are conserved. Gene expression pattern analysis revealed that most TaARFAl genes were relatively highly expressed during different growth and development stages, biotic stress, and abiotic stress, indicating that they might play an important role in development and the stress response. TaARFA1 might interact with PP2C, supporting the role of TaARFA1 genes in the wheat stress response. Taken together, the present study provided comprehensive insights into the structure, organization, evolution, and expression profiles of the TaARF gene family in wheat, which support further functional characterization of TaARF family genes for the development of high-quality wheat varieties.

\section{References}

Altschul SF, Madden TL, Schäffer AA, Zhang J, Zhang Z, Miller W, Lipman DJ. 1997. Gapped BLAST and PSI-BLAST: a new generation of protein database search programs. Nucleic acids research 25 (17):3389-3402 DOI 10.1093/nar/25.17.3389.

Anne-Laure T, Adrien G, Veitia RA. 2014. Transcription factors: specific DNA binding and specific gene regulation. Trends in Genetics 30 (6):211-219 DOI 10.1016/j.tig.2014.04.002.

Anton N, Kateryna D. Makova, Li W-H. 2002. The KA/KS Ratio Test for Assessing the Protein-Coding Potential of Genomic Regions: An Empirical and Simulation Study. Genome Research 12 (1):198-202 DOI 10.1101/gr.200901.

Bailey TL, Williams N, Misleh C, Li WW. 2006. MEME: discovering and analyzing DNA and protein sequence motifs. Nucleic acids research 34:369-373 DOI 10.1093/nar/gk1198.

Bajwa AA, Farooq M, Nawaz A. 2018. Seed priming with sorghum extracts and benzyl 
445

446

447

448

449

450

451

452

453

454

455

456

457

458

459

460

461

462

463

464

465

466

467

468

469

470

471

472

473

474

475

476

477

478

479

480

481

482

483

484

aminopurine improves the tolerance against salt stress in wheat (Triticum aestivum L.). Physiology and Molecular Biology of Plants 24 (2):239-249 DOI 10.1007/s12298-0180512-9.

Bérauddufour S, Paris S, Chabre M, Antonny B. 1999. Dual Interaction of ADP Ribosylation Factor 1 with Sec7 Domain and with Lipid Membranes during Catalysis of Guanine Nucleotide Exchange. Journal of Biological Chemistry 274 (53):37629-37636 DOI 10.1074/jbc.274.53.37629.

Boman AL, Zhang Cj, Zhu X, Kahn RA. 2000. A family of ADP-Ribosylation factor effectors that can alter membrane transport through the trans-Golgi. Mol biol cell 11 (4):1241-1255 DOI 10.1127/0372-8854/2007/0051-0427.

Bourgoin SG, Azreq M-AE. 2012. Small inhibitors of ADP-ribosylation factor activation and function in mammalian cells. World Journal of Pharmacology 1 (4):55-64 DOI 10.5497/wjp.v1.i4.55.

Burgoyne RD. 2001. Small GTP-binding proteins. International Review of Cytology 133 (10):187-230 DOI 10.1016/0968-0004(89)90281-8.

Chen C, Chen H, He Y, Xia R. 2018. TBtools, a Toolkit for Biologists integrating various HTS-data handling tools with a user-friendly interface. Biorxiv DOI 10.1101/289660.

Chen T, Li W, Hu X, Guo J, Liu A, Zhang B. 2015. A Cotton MYB Transcription Factor, GbMYB5, is Positively Involved in Plant Adaptive Response to Drought Stress. Plant and Cell Physiology 56 (5):917-929 DOI 10.1093/pcp/pcv019.

Cole T, Adam R, Loyal G, Geo P, Daehwan K, Kelley DR, Harold P, Salzberg SL, Rinn JL, Lior P. 2012. Differential gene and transcript expression analysis of RNA-seq experiments with TopHat and Cufflinks. Nature protocols 7 (3):562-578 DOI 10.1038/nprot.2012.016.

Conant GC, Wolfe KH. 2008. Turning a hobby into a job: How duplicated genes find new functions. Nature Reviews Genetics 9 (12):938-950 DOI 10.1038/nrg2482.

Fang Z, Jiang W, He Y, Ma D, Liu Y, Wang S, Zhang Y, Yin J. 2020. Genome-Wide Identification, Structure Characterization, and Expression Profiling of Dof Transcription Factor Gene Family in Wheat (Triticum aestivum L.). Agronomy 10:294 DOI 10.3390/agronomy10020294.

Finn RD, Mistry J, Schuster-Böckler B, Sam GJ, Volker H, Timo L, Simon M, Mhairi M, Ajay K, Richard D. 2006. Pfam: clans, web tools and services. Nucleic acids Research 34:247-251 DOI 10.1093/nar/gkj149.

Gebbie LK, Burn JE, Hocart CH, Williamson RE. 2005. Genes encoding ADP-ribosylation factors in Arabidopsis thaliana L. Heyn.; genome analysis and antisense suppression. Journal of Experimental Botany 56 (414):1079-1091 DOI 10.1093/jxb/eri099.

Gu Z, Cavalcanti A, Chen FC, Bouman P, Li WH. 2002. Extent of Gene Duplication in the Genomes of Drosophila, Nematode, and Yeast. Molecular Biology and Evolution 19 (3):256-262 DOI 10.1093/oxfordjournals.molbev.a004079.

Holub EB. 2001. The arms race is ancient history in Arabidopsis, the wildflower. Nature reviews Genetics 2 (7):516-527 DOI 10.1038/35080508. 
485

486

487

488

489

490

491

492

493

494

495

496

497

498

499

500

501

502

503

504

505

506

507

508

509

510

511

512

513

514

515

516

517

518

519

520

521

522

523

524

Horton P, Park K, Obayashi T, Nakai K .2006. Protein subcellular localization prediction with WoLF PSORT. In: Asian Pacific Bioinformatics Conference pp: $39-48$ DOI 10.1142/97818609472920007.

Hu B, Jin J, Guo AY, Zhang H, Luo J, Gao G. 2015. GSDS 2.0: an upgraded gene feature visualization server. Bioinformatics 31 (8):1296-1297 DOI 10.1093/bioinformatics/btu817.

Ivy W, Ines L, Gerit B, Lennart E-L, Hoang-Hoa L, Naumann K, Dangl JL, Scheel D, Lee J. 2010. A protein phosphatase $2 \mathrm{C}$, responsive to the bacterial effector AvrRpm1 but not to the AvrB effector, regulates defense responses in Arabidopsis. The Plant Journal 61 (2):249-258 DOI 10.1111/j.1365-313x.2009.04047.x.

Jessica A Schlueter, Jer-Young Lin, Shannon D Schlueter, Iryna F Vasylenko-Sanders, Shweta Deshpande, Jing Yi, Majesta O'Bleness, Bruce A Roe, Rex T Nelson, Brian E Scheffler, Jackson SA, Shoemaker RC. 2007. Gene duplication and paleopolyploidy in soybean and the implications for whole genome sequencing. BMC Genomics 8 (1):330 DOI 10.1186/1471-2164-8-330.

Jiang W, Yang L, He Y, Zhang H, Li W, Chen H, Ma D, Yin J. 2019. Genome-wide identification and transcriptional expression analysis of superoxide dismutase (SOD) family in wheat. PeerJ 7 (2):e8062 DOI 10.7717/peerj.8062.

Joshi R, Ramanarao MV, Lee S, Kato N, Baisakh N. 2014. Ectopic expression of ADP ribosylation factor 1 (SaARF1) from smooth cordgrass (Spartina alterniflora Loisel) confers drought and salt tolerance in transgenic rice and Arabidopsis. Plant Cell, Tissue and Organ Culture 117 (1):17-30 DOI 10.1007/s11240-013-0416-X.

K Meyer, MP Leube, Grill E. 1994. A protein phosphatase $2 \mathrm{C}$ involved in ABA signal transduction in Arabidopsis thaliana. Science 264 (5164):1452-1455 DOI 10.1126/science.8197457.

Kazuo Shinozaki, Yamaguchi-Shinozaki K. 2006. Gene networks involved in drought stress response and tolerance. Journal of Experimental Botany 58 (2):221-227 DOI 10.1093/jxb/erl164.

Kolde R. 2015. pheatmap: Pretty Heatmaps.

Kumar S, Stecher G, Tamura K. 2016. MEGA7: Molecular Evolutionary Genetics Analysis Version 7.0 for Bigger Datasets. Molecular Biology and Evolution 33 (7):1870-1874 DOI 10.1093/molbev/msw054.

Lee WY, Hong JK, Kim C-H, Chun HJ, Park HC, Kim JC, Yun DJ, Chung WS, Lee SH, Lee S, Yeol. 2003. Over-expressed rice ADP-ribosylation factor 1 (RARF1) induces pathogenesis-related genes and pathogen resistance in tobacco plants. Physiologia Plantarum 119 (4):573-581 DOI 10.1046/j.1399-3054.2003.00215.x.

Letunic I, Bork P. 2016. Interactive tree of life (iTOL) v3: an online tool for the display and annotation of phylogenetic and other trees. Nucleic Acids Research 44:242-245 DOI 10.1093/nar/gkw290.

Letunic I, Copley RR, Schmidt S, Ciccarelli FD, Doerks T, Schultz J, Ponting CP, Bork P. 2004. SMART 4.0: towards genomic data integration. Nucleic acids research 32:D142-144 
525

526

527

528

529

530

531

532

533

534

535

536

537

538

539

540

541

542

543

544

545

546

547

548

549

550

551

552

553

554

555

556

557

558

559

560

561

562

563

564

DOI 10.1093/nar/gkh088.

Li. Y, Kelly. WG, Jr. JML, Schurko AM, Harfe BD, Hill-Harfe KL, Kahn RA. 2004. Functional genomic analysis of the ADP-ribosylation factor family of GTPases: phylogeny among diverse eukaryotes and function in C. elegans. The FASEB journal: official publication of the Federation of American Societies for Experimental Biology 18 (15):18341850 DOI 10.1096/fj.04-2273com.

Liu L, Hu X, Song J, Zong X, Li D, Li D. 2009. Over-expression of a Zea mays L. Protein phosphatase $2 \mathrm{c}$ gene (ZmPP2C) in Arabidopsis thaliana decreases tolerance to salt and drought. Journal of plant physiology 166 (5):531-542 DOI 10.1016/j.jplph.2008.07.008.

Ma S, Bohnert HJ. 2007. Integration of Arabidopsis thalianastress-related transcript profiles, promoter structures, and cell-specific expression. Genome Biology 8 (4):R49 DOI 10.1186/gb-2007-8-4-r49.

Magali L, Patrice D, Gert T, Kathleen M, Yves M, Yves VdP, Pierre R, Stephane R. 2002. PlantCARE, a database of plant cis-acting regulatory elements and a portal to tools for in silico analysis of promoter sequences. Nucleic Acids Research 30 (1):325-327 DOI 10.1093/nar/30.1.325.

Mi Hee L, Myung Ki M, Yong Jik L, Jing Bo J, Dong Han S, Dae Heon K, Kwang-Hee L, Inhwan H. 2002. ADP-ribosylation factor 1 of Arabidopsis plays a critical role in intracellular trafficking and maintenance of endoplasmic reticulum morphology in Arabidopsis. Plant physiology 129 (4):1507-1520 DOI 10.1104/pp.003624.

Mulder N, Apweiler R. 2007. InterPro and InterProScan: tools for protein sequence classification and comparison. Methods in molecular biology 396:59-70 DOI 10.1007/9781-59745-515-2 5.

Muthamilarasan M, Mangu VR, Zandkarimi H, Prasad M, Baisakh N. 2016. Structure, organization and evolution of ADP-ribosylation factors in rice and foxtail millet, and their expression in rice. Scientific Reports 6 (1):24008 DOI 10.1038/srep24008.

Myung Ki M, Mihue J, Myounghui L, Junho L, Kyungyoung S, Yongjik L, Kwan Yong C, Robinson DG, Inhwan H. 2013. Recruitment of Arf1-GDP to Golgi by Glo3p-type ArfGAPs is crucial for golgi maintenance and plant growth. Plant Physiology 161 (2):676691 DOI 10.1104/pp.112.209148.

Nguyen NH, Jung C, Cheong J-J. 2019. Chromatin remodeling for the transcription of type 2C protein phosphatase genes in response to salt stress. Plant Physiology and Biochemistry 141:325-331 DOI 10.1016/j.plaphy.2019.06.012.

Nussbaumer T, Warth B, Sharma S, Ametz C, Bueschl C, Parich A, Pfeifer M, Siegwart G, Steiner B, Lemmens M, Schuhmacher R, Buerstmayr H, Mayer KFX, Kugler KG, Schweiger W. 2015. Joint Transcriptomic and Metabolomic Analyses Reveal Changes in the Primary Metabolism and Imbalances in the Subgenome Orchestration in the Bread Wheat Molecular Response to Fusarium graminearum. G3: Genes, Genomes, Genetics 5 (12):2579-2592 DOI 10.1534/g3.115.021550.

Oliver T, Schmidt B, Nathan D, Clemens R, Maskell D. 2005. Using reconfigurable hardware 
565

566

567

568

569

570

571

572

573

574

575

576

577

578

579

580

581

582

583

584

585

586

587

588

589

590

591

592

593

594

595

596

597

598

599

600

601

602

603

604

to accelerate multiple sequence alignment with ClustalW. Bioinformatics 21 (16):3431-3432 DOI 10.1093/bioinformatics/bti508.

Ramírez-González RH, Borrill P, D. Lang SAH, J. Brinton, L. Venturini, M. Davey JJ, F. van Ex AP, Y. Khedikar SJR, A. T. Cory TF, L. Concia. 2018. The transcriptional landscape of polyploid wheat. Science 361 (6403):662 DOI 10.1126/science.aar6089.

Rozas J. 2009. DNA Sequence Polymorphism Analysis Using DnaSP. Methods in Molecular Biology 537:337-350 DOI 10.1007/978-1-59745-251-9_17.

Santoshkumar M, Urbi B, Priyadharshini M, Doddabhimappa G, Rajasekar R. 2013. Gene duplication as a major force in evolution. Journal of genetics 92 (1):155-161 DOI 10.1007/s12041-013-0212-8.

Shiferaw B, Smale M, Braun HJ, Duveiller E, Muricho G. 2013. Crops that feed the world 10. Past successes and future challenges to the role played by wheat in global food security. Food Security 5 (3):291-317 DOI 10.1007/s12571-013-0263-y.

Song J, Ma D, Yin J, Yang L, He Y, Zhu Z, Tong H, Chen L, Zhu G, Liu Y, Gao C. 2019. Genome-Wide Characterization and Expression Profiling of Squamosa Promoter Binding Protein-like (SBP) Transcription Factors in Wheat(Triticum aestivum). Agronomy 9 (9):527 DOI 10.3390/agronomy9090527.

Sreenivasuluab N, Harshavardhana VT, Govinda G, Seilera C, Kohli A. 2012. Contrapuntal role of ABA: does it mediate stress tolerance or plant growth retardation under long-term drought stress? Gene 506 (2):265-273 DOI 10.1016/j.gene.2012.06.076.

Takayuki A, Naoki T, Guangxiao Y, Nagao H, Komatsu S. 2005. Genome-wide identification of the rice calcium-dependent protein kinase and its closely related kinase gene families: comprehensive analysis of the CDPKs gene family in rice. Plant Cell Physiology 46 (2):356366 DOI 10.1093/pcp/pci035.

Veraverbeke WS, Delcour JA. 2002. Wheat Protein Composition and Properties of Wheat Glutenin in Relation to Breadmaking Functionality. Critical reviews in food science and nutrition 42 (3):179-208 DOI 10.1080/10408690290825510.

Victoria E P, F Bryan P. 2002. Splitting pairs: the diverging fates of duplicated genes. Nature reviews Genetics 3 (11):827-837 DOI 10.1038/nrg928.

Wang M, Tan Y, Cai C, Zhang B. 2018. Identification and expression analysis of phosphatidy ethanolamine-binding protein (PEBP) gene family in cotton. Genomics 111 (6): 1373-1380 DOI 10.1016/j.ygeno.2018.09.009.

Wang Z, Gerstein M, Snyder M. 2009. RNA-Seq: a revolutionary tool for transcriptomics. Nature Reviews Genetics 10 (1):57-63 DOI 10.1038/nrg2484.

Widholm JM, Chinnala AR, Ryu JH, Song HS, Eggett T, Brotherton JE. 2001. Glyphosate selection of gene amplification in suspension cultures of 3 plant species. Physiologia Plantarum 112 (4):540-545 DOI 10.1034/j.1399-3054.2001.1120411.x.

Wilkins MR, Gasteiger E, Bairoch A, Sanchez JC, Williams KL, Appel RD, Hochstrasser DF. 1999. Protein Identification and Analysis Tools in the ExPASy Server. Methods in Molecular Biology 112:531-552 DOI 10.1385/1-59259-890-0:571. 
605

606

607

608

609

610

611

612

613

614

615

616

617

618

619

620

621

622

623

624

625

626

627

628

629

630

631

632

633

Wonderlich ER, Leonard JA, Kulpa DA, Leopold KE, Norman JM, Collins KL. 2011. ADP ribosylation factor 1 activity is required to recruit AP-1 to the major histocompatibility complex class I (MHC-I) cytoplasmic tail and disrupt MHC-I trafficking in HIV-1-infected primary T cells. Journal of virology 85 (23):12216-12226 DOI 10.1128/JVI.00056-11.

Yin J, Fang Z, Sun C, Zhang P, Zhang X, Chen L, Wang S, Ma D, Zhu Y. 2018. Rapid identification of a stripe rust resistant gene in a space-induced wheat mutant using specific locus amplified fragment (SLAF) sequencing. Scientific Reports 8 (1):3086 DOI 10.1038/s41598-018-21489-5.

Yu X, Han J, Li L, Zhang Q, Yang G, He G. 2020. Wheat PP2C-a10 regulates seed germination and drought tolerance in transgenic Arabidopsis. Plant Cell Reports 39 (5):635651 DOI 10.1007/s00299-020-02520-4.

Yuzuki Manabe, Ray A. Bressan, Tao Wang, Fang Li, Hisashi Koiwa, Irina Sokolchik, Xia Li, Maggio A. 2007. The Arabidopsis Kinase-Associated Protein Phosphatase Regulates Adaptation to Na+ Stress. Plant Physiology 146 (2):612-622 DOI 10.1104/pp.107.109009.

Zengcui Z, Harry B, Piotr G, Mathieu C, Jérémy J, Cécile H, Ghislaine M, Arnaud C, Sylvie S, Bikram S G, Jack B R, Valérie B, Justin D F, Boulos C . 2011. Duplication and partitioning in evolution and function of homoeologous $\mathrm{Q}$ loci governing domestication characters in polyploid wheat. Proceedings of the National Academy of Sciences of the United States of America 108 (46):18737-18742 DOI 10.1073/pnas.1110552108.

Zhang Z, Jun L, Xiao-Qian Z, Jun W, Wong GK-S, Jun Y. 2006. Kaks_calculator: calculating ka and ks through model selection and model averaging. Genomics Proteomics Bioinformatics 4 (4):259-263 DOI 10.1016/S1672-0229(07)60007-2.

Zhao J, Zhai Z, Li Y, Geng S, Song G, Guan J, Jia M, Wang F, Sun G, Feng N, Kong X, Chen L, Mao L, Li A. 2018. Genome-Wide Identification and Expression Profiling of the TCP Family Genes in Spike and Grain Development of Wheat (Triticum aestivum L.). Frontiers in Plant Science 9:1282 DOI 10.3389/fpls.2018.01282.

Zuguang G, Lei G, Roland E, Matthias S, Benedikt B. 2014. circlize Implements and enhances circular visualization in R. Bioinformatics 30 (19):2811-2812 DOI 10.1093/bioinformatics/btu393.

Peer) reviewing PDF | (2020:10:54615:1:1:NEW 30 Dec 2020) 
Table $\mathbf{1}$ (on next page)

ARF transcription factor families in wheat 
Table 1. ARF transcription factor families in wheat.

\begin{tabular}{|c|c|c|c|c|c|c|c|}
\hline Gene Name & Accession numbers & Location & Exons & Introns & Length & MW & pI \\
\hline TaARFAla-A & TraesCS1A02G306200.1 & chr1A:498451494-498454124 & 5 & 4 & 186 & 21.32 & 6.92 \\
\hline TaARFAla-B & TraesCS1B02G317000.1 & chr1B:541466983-541469854 & 6 & 5 & 184 & 21.11 & 6.97 \\
\hline TaARFAla-D & TraesCS1D02G305900.1 & chr1D:403235691-403239527 & 6 & 6 & 263 & 29.64 & 9.35 \\
\hline TaARFAIb-A & TraesCS2A02G235500.1 & chr2A:293934939-293967206 & 6 & 5 & 181 & 20.57 & 6.43 \\
\hline TaARFAlc-D & TraesCS2D02G244900.1 & chr2D:277672480-277681787 & 5 & 6 & 181 & 20.58 & 6.43 \\
\hline TaARFAld-A & TraesCS3A02G337300.1 & chr3A:584275968-584279016 & 6 & 7 & 182 & 20.88 & 6.43 \\
\hline TaARFAld-B & TraesCS3B02G368600.1 & chr3B:581026448-581031825 & 6 & 5 & 181 & 20.71 & 6.43 \\
\hline TaARFAld-D & TraesCS3D02G330500.2 & chr3D:443258251-443265348 & 6 & 5 & 181 & 20.71 & 6.43 \\
\hline TaARFAle-A & TraesCS5A02G142100.1 & chr5A:315494544-315497231 & 5 & 5 & 181 & 20.44 & 6.09 \\
\hline TaARFAle-B & TraesCS5B02G140900.1 & chr5B:266003993-266006657 & 5 & 5 & 181 & 20.44 & 6.09 \\
\hline TaARFAle-D & TraesCS5D02G150000.1 & chr5D:238710757-238712733 & 5 & 4 & 183 & 20.65 & 6.09 \\
\hline TaARFAlf-A & TraesCS5A02G467400.1 & chr5A:645260990-645263277 & 6 & 5 & 211 & 24.02 & 7.77 \\
\hline TaARFAIf-B & TraesCS5B02G479100.1 & chr5B:650545995-650548824 & 5 & 5 & 211 & 24.02 & 6.02 \\
\hline TaARFAlf-D & TraesCS5D02G480200.1 & chr5D:517840384-517842860 & 6 & 5 & 211 & 23.99 & 7.77 \\
\hline TaARFBIa-A & TraesCS1A02G197800.1 & chr1A:355714313-355716842 & 6 & 5 & 191 & 21.55 & 6.3 \\
\hline TaARFBIa-B & TraesCS1B02G212500.1 & chr1B:386159117-386161564 & 6 & 5 & 191 & 21.55 & 6.3 \\
\hline TaARFBIa-D & TraesCS1D02G201300.1 & chr1D:284239314-284241950 & 6 & 5 & 191 & 21.55 & 6.3 \\
\hline TaARFBIb-A & TraesCS6A02G086900.1 & chr6A:55551759-55555137 & 6 & 6 & 194 & 21.82 & 5.58 \\
\hline TaARFBIC-A & TraesCS6A02G268000.1 & chr6A:494440084-494443530 & 6 & 5 & 191 & 21.37 & 6.83 \\
\hline TaARFBId-B & TraesCS6B02G114900.1 & chr6B:98857984-98861271 & 5 & 5 & 172 & 19.45 & 5.81 \\
\hline TaARFBIc-B & TraesCS6B02G295200.1 & chr6B:530127311-530130936 & 6 & 5 & 246 & 27.97 & 9.3 \\
\hline TaARFB1c-D & TraesCS6D02G246900.1 & chr6D:349986601-349990113 & 6 & 5 & 191 & 21.40 & 6.83 \\
\hline TaARFBIe- $N$ & TraesCSU02G019600.1 & chrUn:21195216-21198738 & 6 & 6 & 194 & 21.84 & 5.82 \\
\hline TaARFC1-A & TraesCS4A02G149200.1 & chr4A:284629181-284631736 & 2 & 1 & 184 & 20.38 & 5.94 \\
\hline TaARFC1-B & TraesCS4B02G164400.1 & chr4B:340990997-340999096 & 2 & 2 & 184 & 20.31 & 5.84 \\
\hline TaARF3-A & TraesCS7A02G284000.1 & chr7A:319587234-319598812 & 8 & 7 & 182 & 20.31 & 5.73 \\
\hline
\end{tabular}




\begin{tabular}{|c|c|c|c|c|c|c|c|}
\hline TaARF3-B & TraesCS7B02G181400.1 & chr7B:274309305-274319048 & 8 & 7 & 198 & 22.29 & 6.14 \\
\hline TaARF3-D & TraesCS7D02G282400.1 & chr7D:287897903-287906900 & 8 & 7 & 198 & 22.29 & 5.46 \\
\hline TaARFC1-D & TraesCS4D02G152600.1 & chr4D:185795832-185798286 & 2 & 2 & 184 & 20.35 & 6.05 \\
\hline TaARLIa-A & TraesCS3A02G172900.1 & chr3A:189275871-189280985 & 3 & 2 & 193 & 21.94 & 5.95 \\
\hline TaARLIa-B & TraesCS3B02G203800.1 & chr3B:237643721-237648261 & 3 & 2 & 193 & 21.94 & 5.95 \\
\hline TaARL1a-D & TraesCS3D02G179300.1 & chr3D:160908142-160912382 & 3 & 2 & 201 & 22.86 & 6.32 \\
\hline TaARLIb- $A$ & TraesCS3A02G193800.1 & chr3A:266450899-266454547 & 3 & 2 & 193 & 22.11 & 6.97 \\
\hline TaARLIb-B & TraesCS3B02G221700.1 & chr3B:276848921-276852339 & 3 & 2 & 193 & 22.11 & 6.97 \\
\hline TaARL1b-D & TraesCS3D02G196200.1 & chr3D:193594920-193598371 & 3 & 2 & 193 & 22.13 & 6.97 \\
\hline TaARLIC-A & TraesCS4A02G045000.1 & chr4A:37062663-37067415 & 5 & 4 & 184 & 20.73 & 9.15 \\
\hline TaARL1c-B & TraesCS4B02G260300.1 & chr4B:527276119-527280600 & 5 & 4 & 184 & 20.73 & 9.15 \\
\hline TaARL1c-D & TraesCS4D02G260100.1 & chr4D:429465149-429469539 & 5 & 4 & 184 & 20.73 & 9.15 \\
\hline TaARL1d-A & TraesCS5A02G089200.1 & chr5A:119438999-119442370 & 3 & 2 & 193 & 22.04 & 6.91 \\
\hline TaARLId-B & TraesCS5B02G095200.1 & chr5B:125228094-125232113 & 3 & 2 & 193 & 22.08 & 7.75 \\
\hline TaARL1d-D & TraesCS5D02G101300.1 & chr5D:114349156-114352814 & 3 & 2 & 193 & 22.06 & 6.91 \\
\hline TaARLIe-A & TraesCS6A02G293900.1 & chr6A:525759292-525762110 & 6 & 5 & 184 & 20.60 & 8.32 \\
\hline TaARL1e-B & TraesCS6B02G324400.1 & chr6B:573689158-573692125 & 6 & 6 & 175 & 19.49 & 8.31 \\
\hline TaARLIe-D & TraesCS6D02G274800.1 & chr6D:383544182-383547096 & 6 & 6 & 184 & 20.60 & 8.32 \\
\hline TaARL1f-B & TraesCS7B02G236300.1 & chr7B:440347979-440349242 & 3 & 2 & 193 & 22.14 & 6.59 \\
\hline TaARL1g-D & TraesCS7D02G332100.1 & chr7D:423583936-423585168 & 3 & 2 & 193 & 22.11 & 6.21 \\
\hline TaGBIa-A & TraesCS1A02G069400.1 & chr1A:52056090-52060042 & 6 & 6 & 203 & 22.93 & 5.67 \\
\hline$T a G B 1 b-B$ & TraesCS2B02G191100.1 & chr2B:166468400-166471957 & 6 & 4 & 170 & 19.34 & 5.89 \\
\hline$T a G B 1 c-D$ & TraesCS2D02G172000.1 & chr2D:115947943-115950782 & 7 & 5 & 199 & 22.51 & 7.09 \\
\hline$T a G B 1 a-B$ & TraesCS1B02G087700.1 & chr1B:83206061-83209210 & 6 & 6 & 202 & 22.81 & 6.07 \\
\hline$T a G B 1 a-D$ & TraesCS1D02G072000.1 & chr1D:52358164-52361912 & 6 & 6 & 203 & 22.94 & 5.67 \\
\hline TaTTN5a-A & TraesCS1A02G421700.1 & chr1A:577760728-577762543 & 6 & 5 & 197 & 22.62 & 8.36 \\
\hline TaTTN5b-D & TraesCS1D02G429700.1 & chr1D:481330712-481333368 & 5 & 5 & 185 & 21.17 & 8.46 \\
\hline TaTTN5c-A & TraesCS5A02G014900.1 & chr5A:10476109-10479944 & 5 & 5 & 185 & 21.14 & 8.46 \\
\hline
\end{tabular}




\begin{tabular}{llllllll} 
TaTTN5c-B & TraesCS5B02G013100.1 & chr5B:13174286-13178149 & 5 & 5 & 185 & 21.08 & 8.46 \\
TaTTN5c-D & TraesCS5D02G020800.1 & chr5D:13715586-13718281 & 5 & 4 & 185 & 21.14 & 8.46 \\
\hline
\end{tabular}

2 Length, protein length, aa; $\mathrm{MW}$, molecular weight, $\mathrm{kDa}$; $\mathrm{pI}$, isoelectric point. 


\section{Figure 1}

Phylogenetic relationship of wheat, rice, and Arabidopsis.

Phylogenetic relationship of wheat, rice, and Arabidopsis. Full length amino acid sequences of hexaploid wheat (Ta), Rice (Os), and Arabidopsis (At) were aligned by ClustalW2 and an unrooted neighbor-joining tree was constructed with 1000 bootstrap iterations. The tree classified the proteins into seven distinct classes, shaded in red, orange, yellow, green, purple, blue, and grey. Proteins from rice, Arabidopsis, and wheat are represented in yellow, red, and green closed circles, respectively 


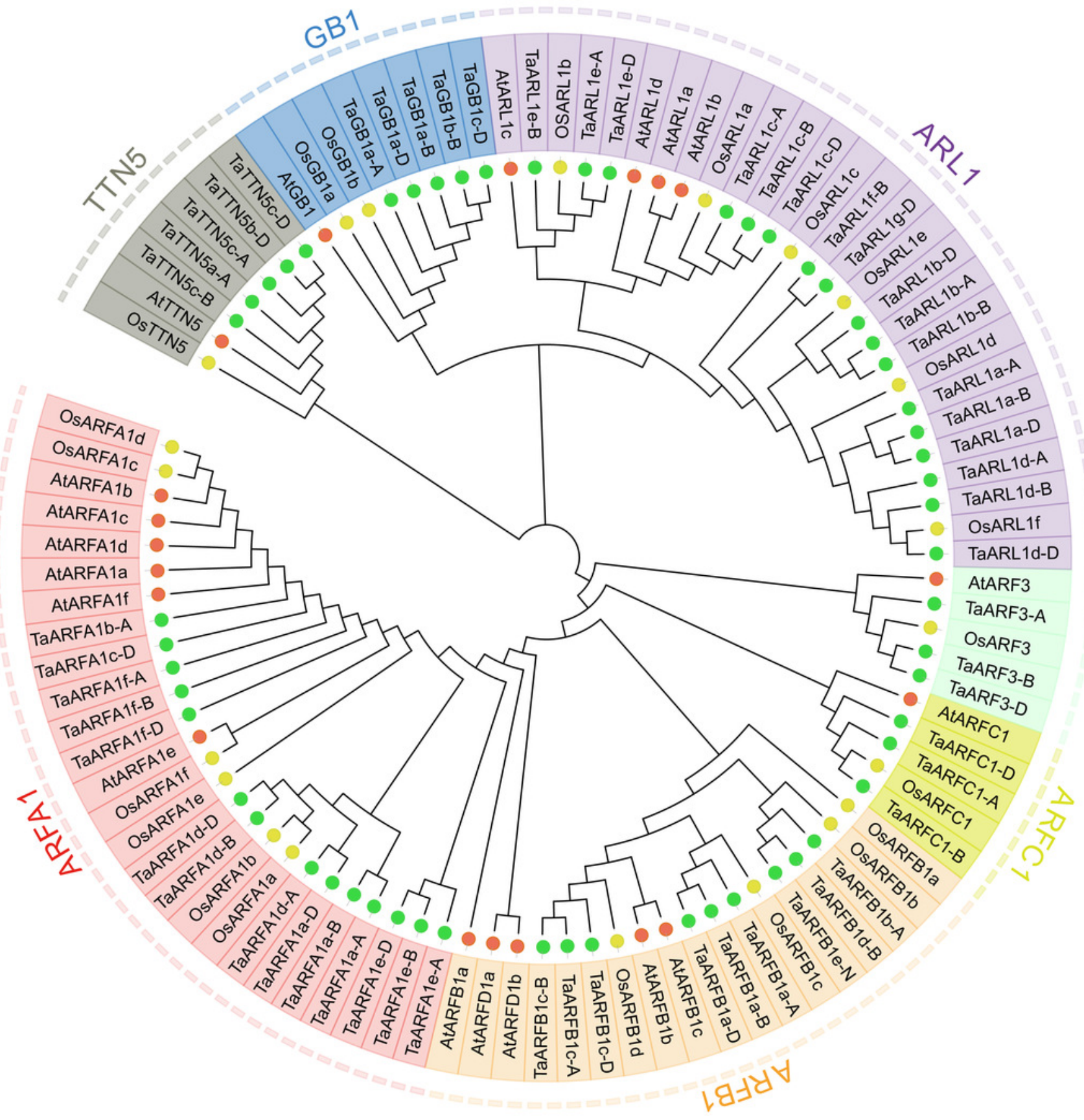




\section{Figure 2}

Comparative analysis of the phylogenetics, exon-intron structures, and conserved motifs of the ARF family in wheat (TaARFs)

Comparative analysis of the phylogenetics, exon-intron structures, and conserved motifs of the ARF family in wheat (TaARFs). (A) The phylogenetic tree of 56 ARF proteins; (B) Gene structures of 56 ARF proteins in hexaploid wheat. The yellow boxes are coding sequences (CDSs), the black lines are introns, and the blue boxes are 5'-untranslated regions (UTRs) or 3'-UTRs; (C) Motif composition models of 56 ARF proteins. Different motifs are color-coded according to the legend.

(A)

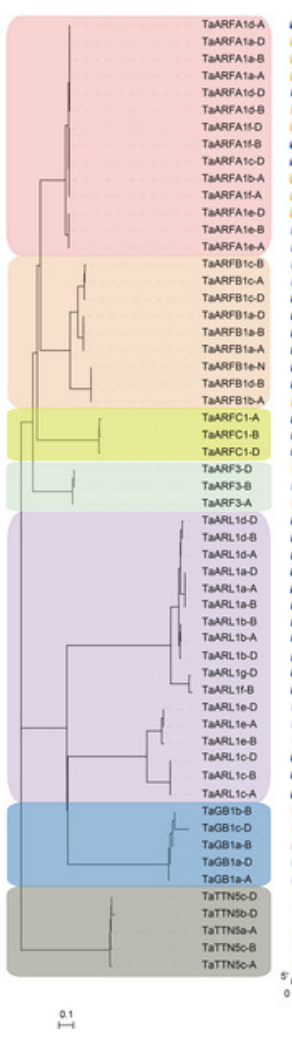

(B)

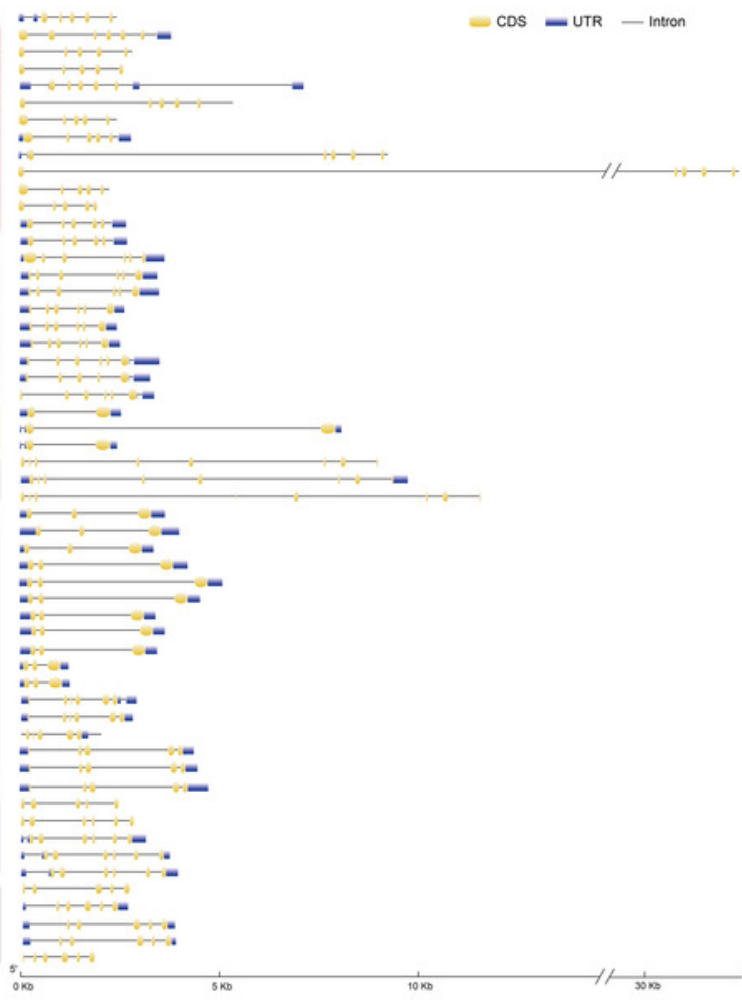

(C)

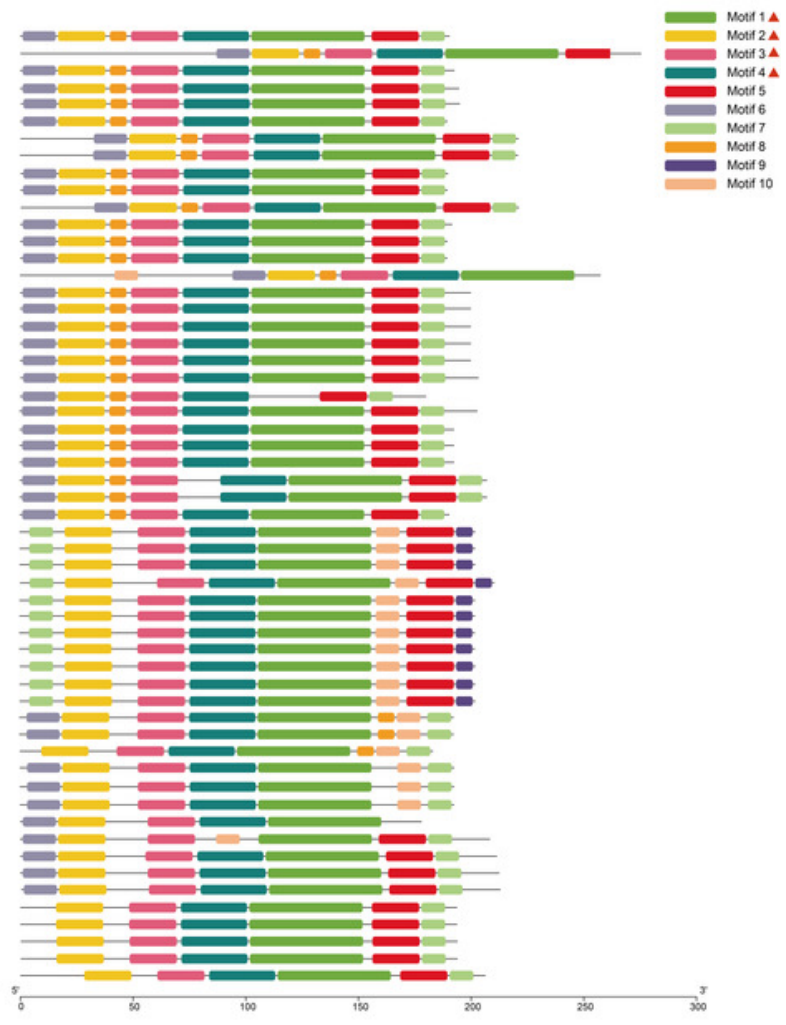




\section{Figure 3}

Chromosomal locations and gene duplication events in TaARFs

Chromosomal locations and gene duplication events in TaARFs. (A): Chromosomal locations of the TaARF genes in wheat. The ruler on the left indicates the physical map distance between genes (Mb). The black dots represent the centromeres. Different groups of TaARFs are represented by different colors. The triads are indicated with gray lines; (B): $\mathrm{Ka} / \mathrm{Ks}$ values for duplicated TaARF gene pairs; (C): Gene duplication events of TaARFs. 
(A)
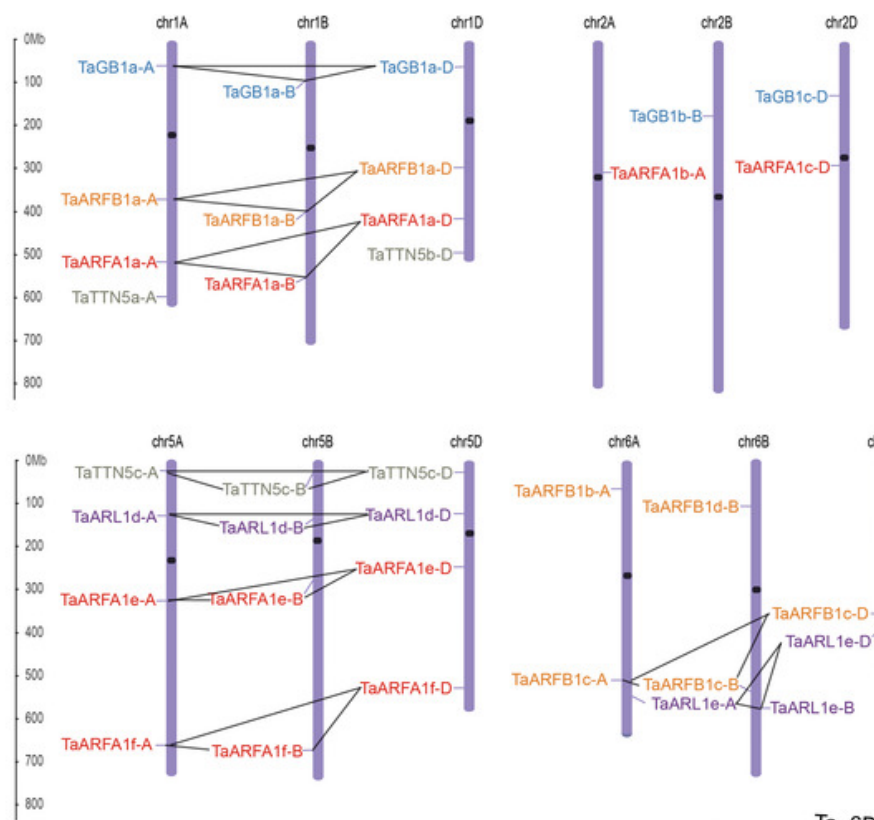
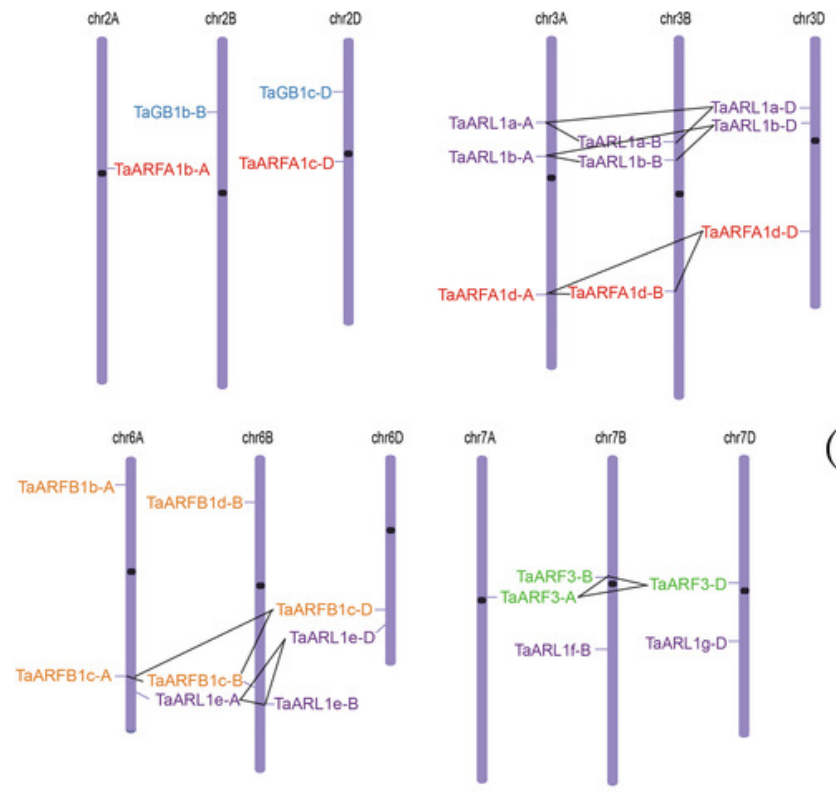

(B)

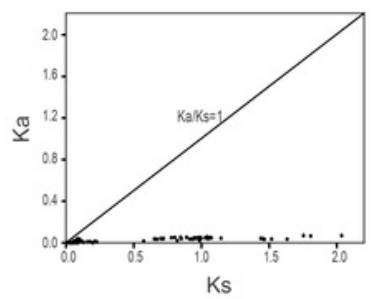

(C)

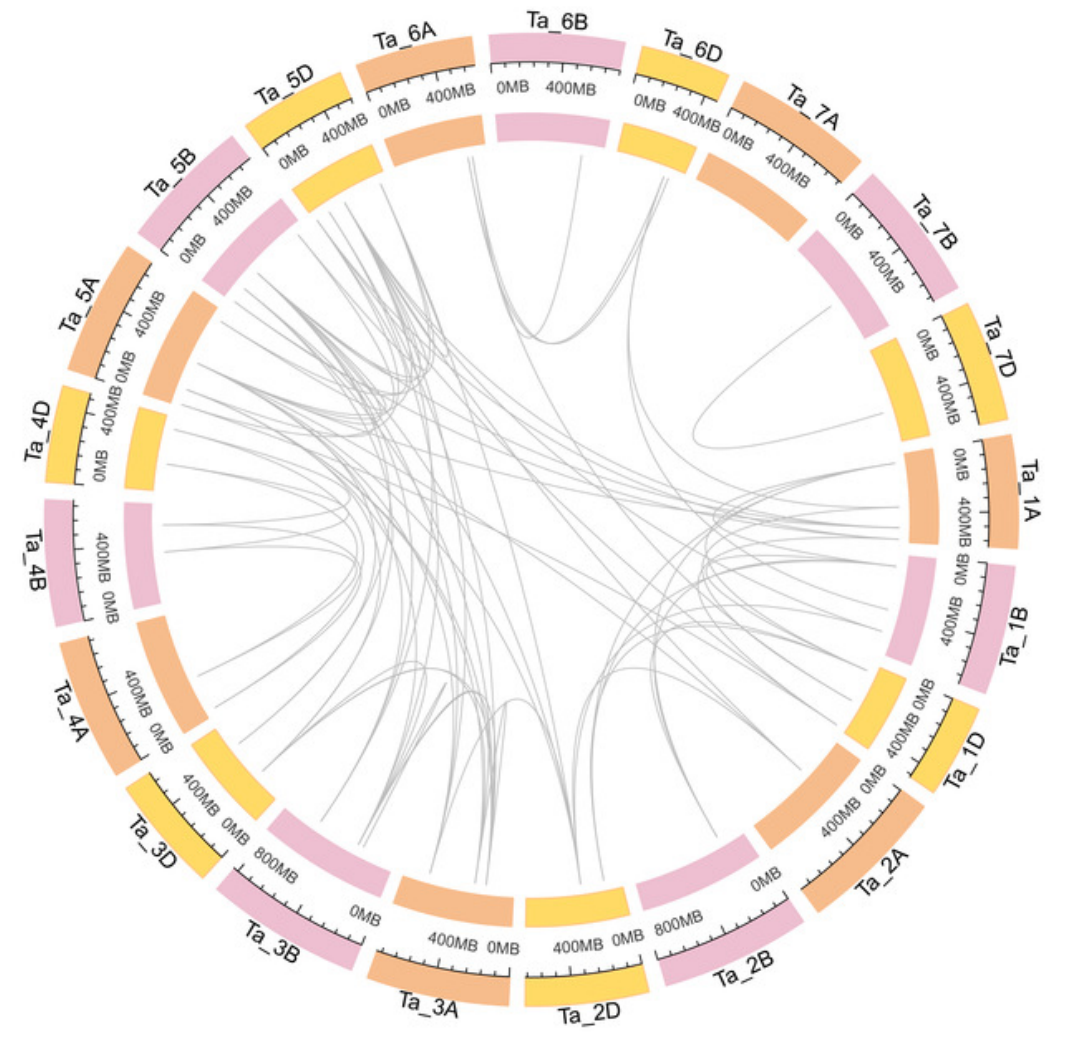




\section{Figure 4}

Gene duplication events of $A R F$ genes between wheat and other plant species.

Gene duplication events of $A R F$ genes between wheat and other plant species. Gray lines in the background indicate the syntenic ARF gene pairs. Colored lines in the background indicate the orthologous genes across the four species, with red indicating the ARFAl supgroup genes and blue indicating the ARL1 sup-group genes. (A): Gene duplication events of $A R F$ genes between wheat and Aegilops tauschii. (B): Gene duplication events of $A R F$ genes between wheat and Arabidopsis. (C): Gene duplication events of $A R F$ genes between wheat and rice. (D): Gene duplication events of $A R F$ genes between wheat and Triticum dicoccoides. 
(A)

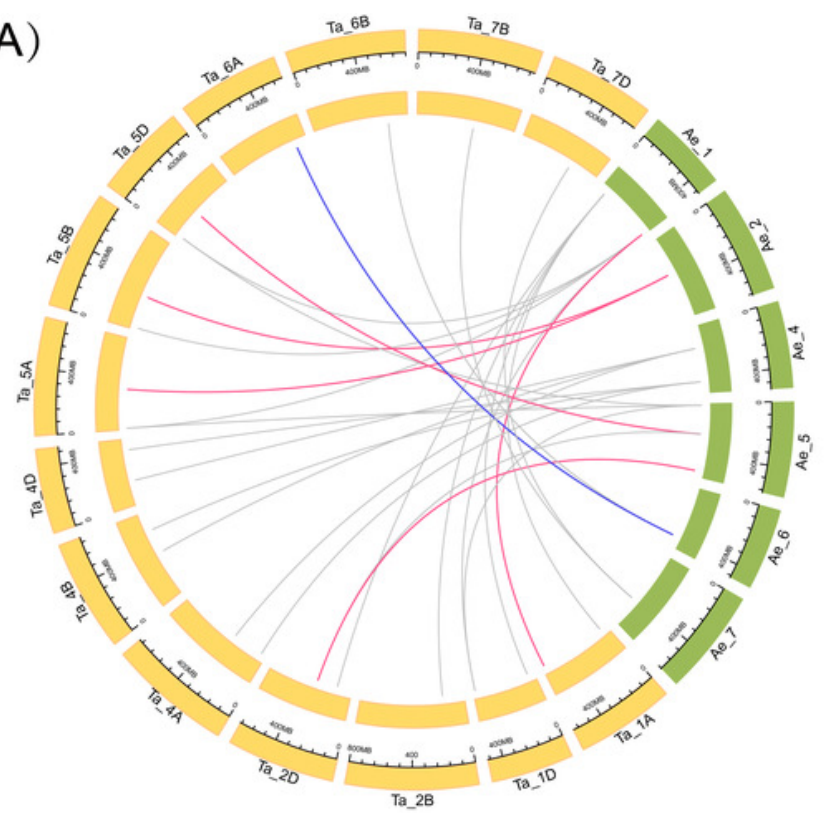

(C)

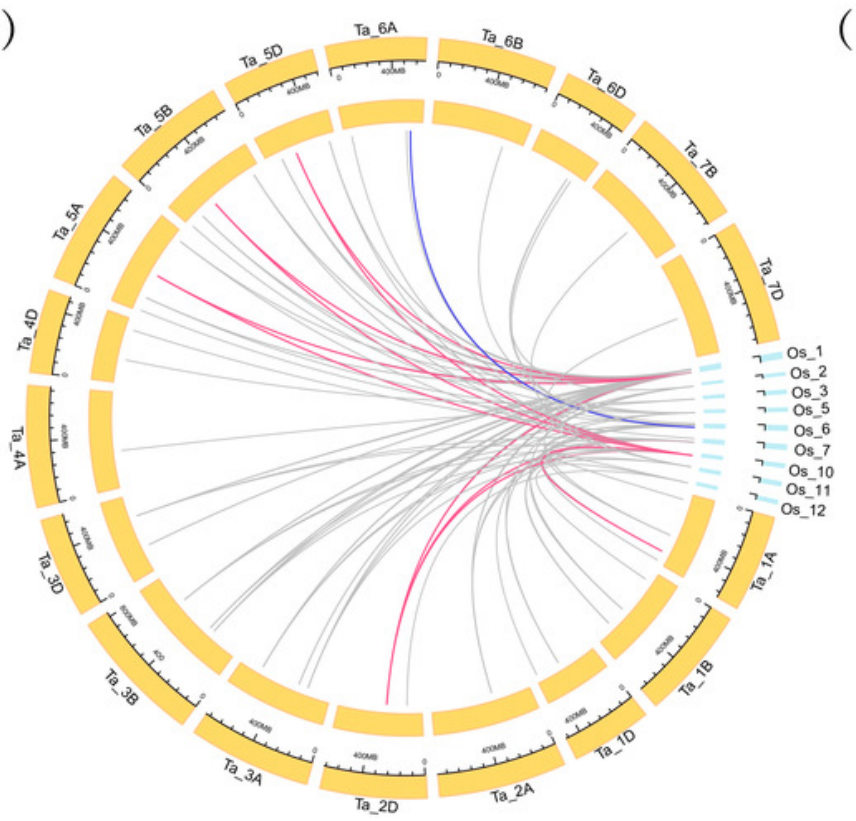

(B)

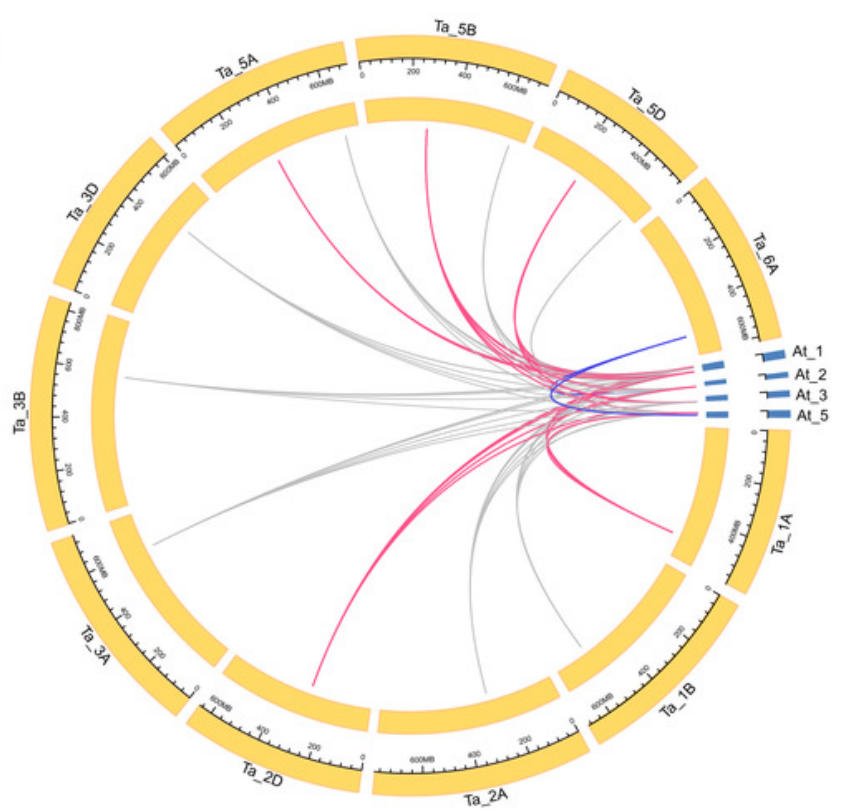

(D)

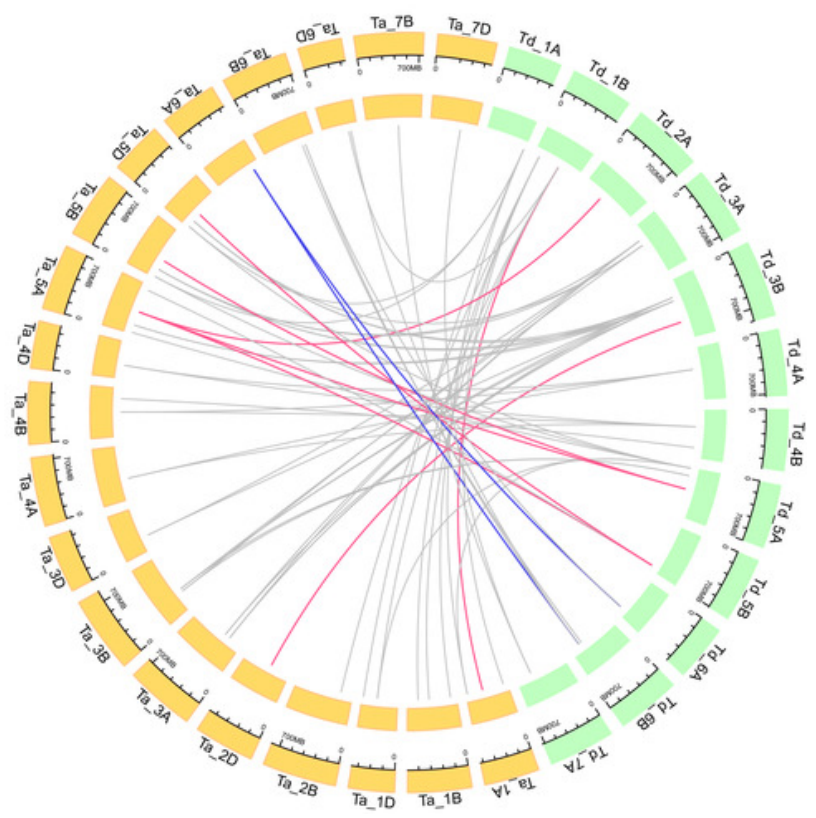




\section{Figure 5}

Expression analysis of TaARF genes in different tissues.

Expression analysis of TaARF genes in different tissues. (A) Heatmap showing the expression levels of TaARF genes in different subfamilies (columns) and wheat developmental stages/tissues (rows); (B) Expression balance for all 1:1:1 triads was plotted in a triangular plot with the coordinates of each circle representing the normalized expression of $A, B$ and $D$ homoeologs. Triads are indicated by circles, with areas separated by gray lines indicating expression patterns that are balanced, dominant for one sub-genome homoeolog, or suppressed for one homoeolog, as previously described. Colored circles represent subgroups; (C) An "electronic fluorescence pictogram" representation of the target TaARFAla-A gene expression pattern based on the Wheat Atlas dataset. 
(A)

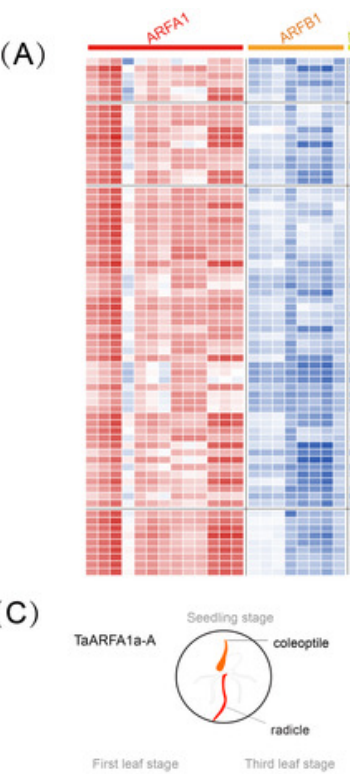

(C)

ith leat stage

ffinh leat
blade
fren leat
sheat

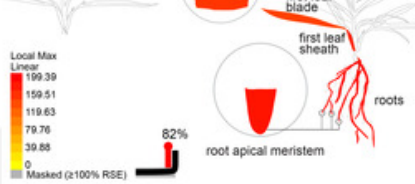

(B)

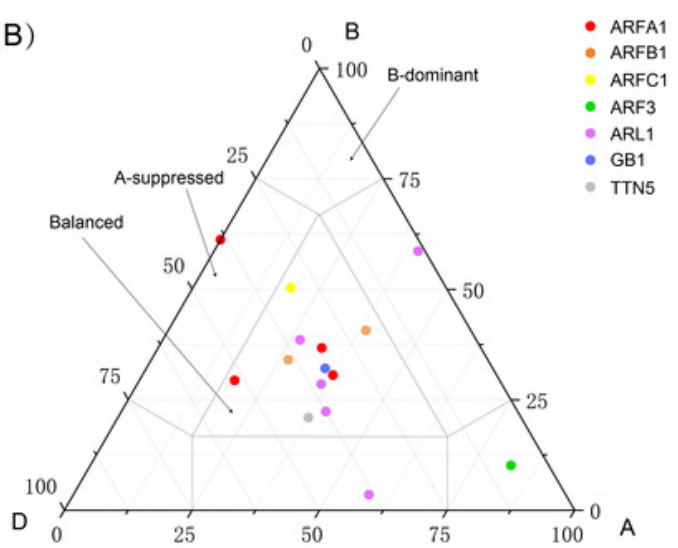

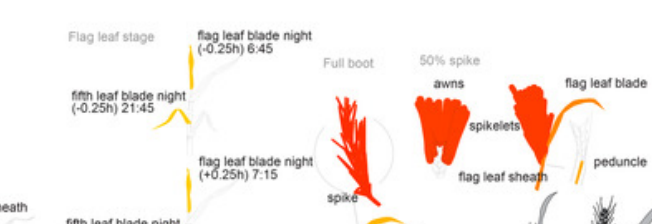

Epikelet amms - forseat

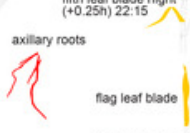

tamileat basec<smiles>C1=I[In]1</smiles><smiles>ICCI</smiles>
irgue<smiles>C1C2CC3C1C3C2</smiles>

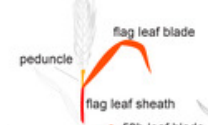

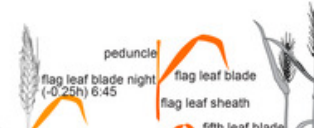

$1 / 1$

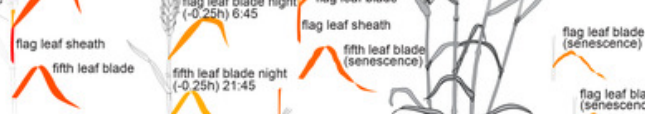

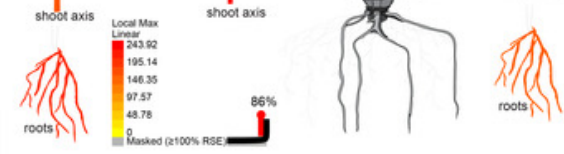

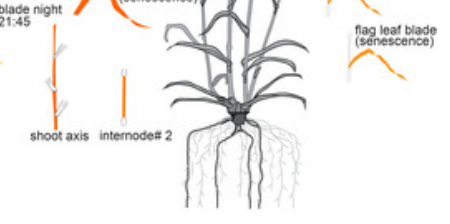




\section{Figure 6}

Expression patterns of $A R F$ genes in wheat (TaARFs) under different abiotic and biotic stresses.

Expression patterns of $A R F$ genes in wheat (TaARFs) under different abiotic and biotic stresses. (A): Expression of TaARFA1 members in the leaf under drought, heat, and drought \& heat combined stress. (B): Expression of TaARFAl members in the leaf under powdery mildew and stripe rust stress. 

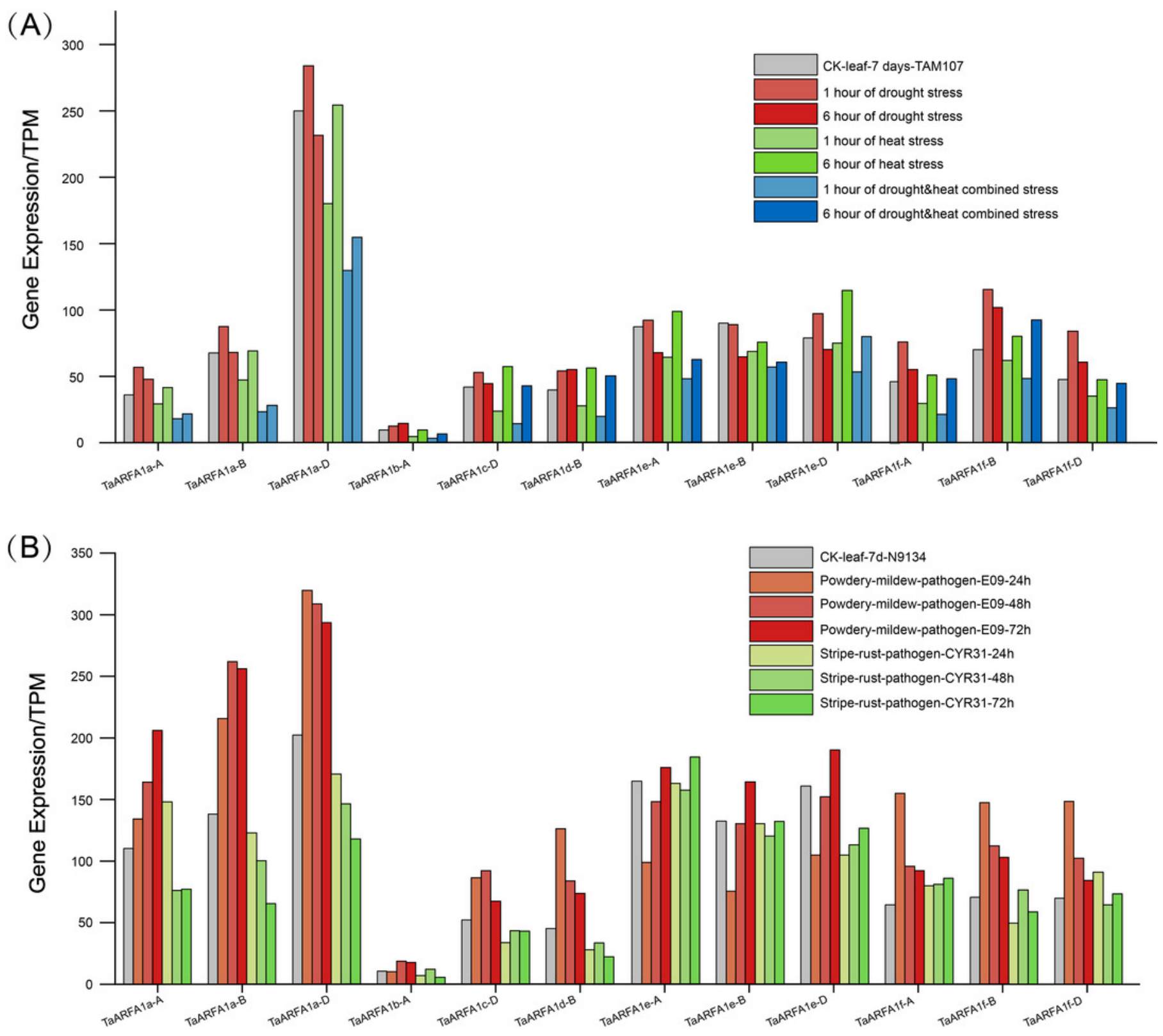
Figure 7

Expression of TaARFA1 members in root under ABA, salt and cold stress.

Expression of TaARFA1 members in root under ABA, salt and cold stress. The roots were sampled after 6 and $12 \mathrm{~h}$ of $\mathrm{ABA}(100 \mu \mathrm{M}), \mathrm{NaCl}(150 \mathrm{mmol})$, and cold $\left(4^{\circ} \mathrm{C}\right)$ treatments. The white line represent the expression levels of control $(*, p<0.05)$.

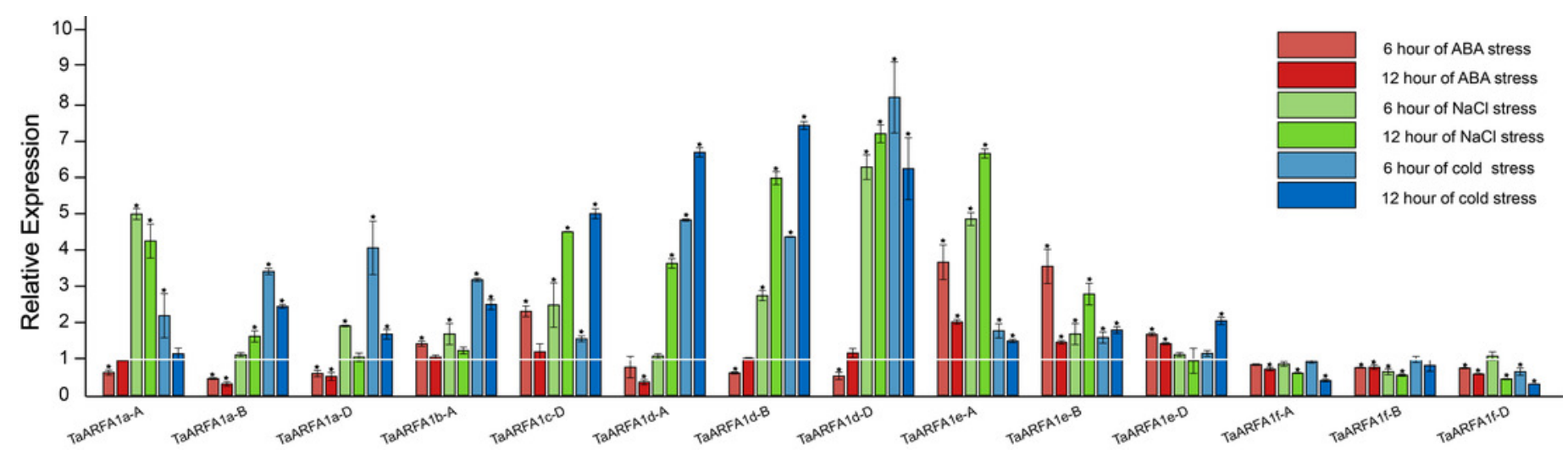


Figure 8

Predicted cis-acting elements in the promoters of TaARF genes.

Predicted cis-acting elements in the promoters of TaARF genes. (A) Cis-acting elements involved in stress in the promoter of TaARF genes. The different colors and numbers of the grid indicate the number of different promoter elements; $(B)$ The number of cis-acting elements in TaARF genes. The histogram represents the sum of the cis-acting elements in each category.

(A)
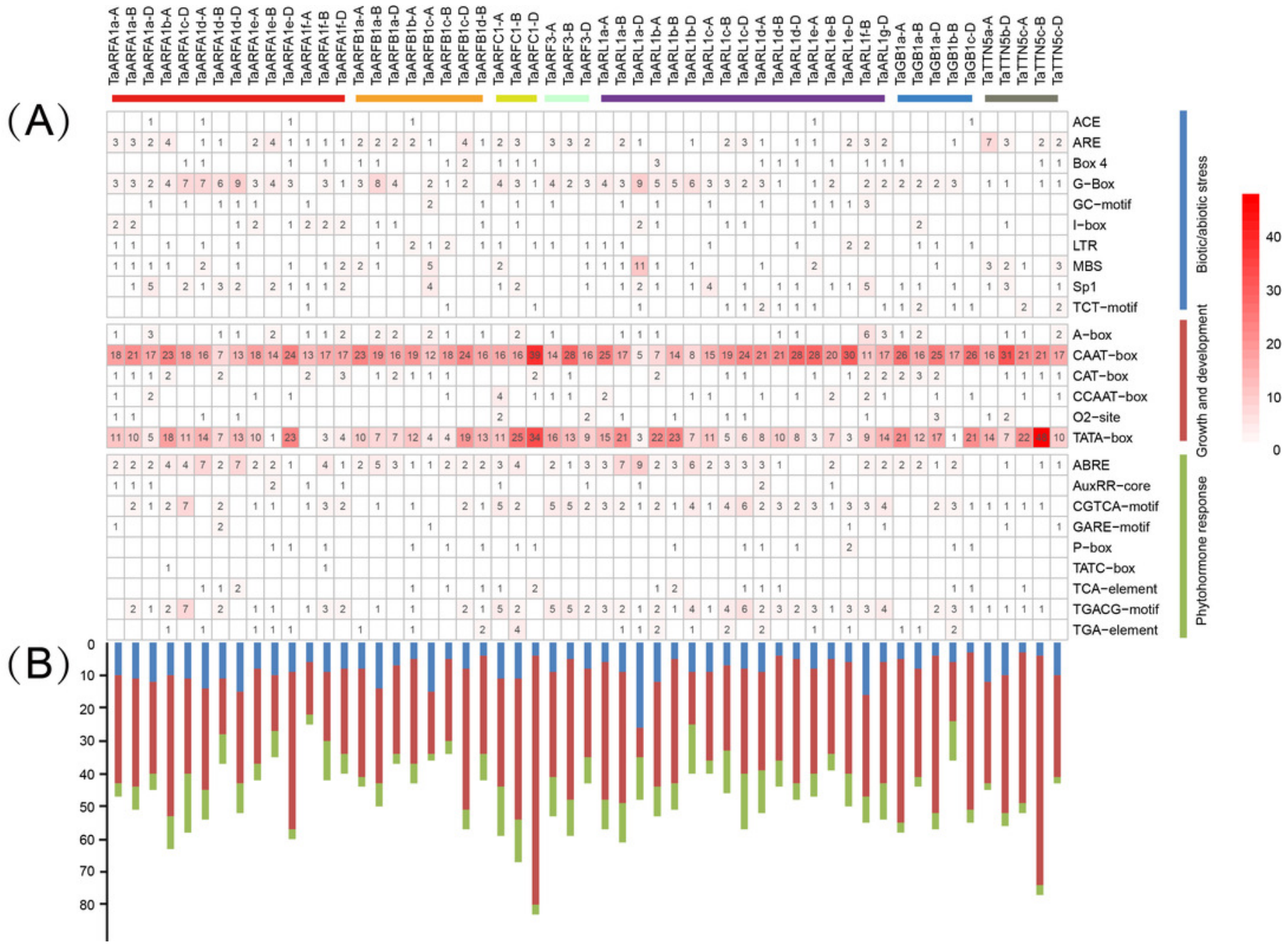


\section{Figure 9}

Gene ontology annotation and protein interaction network of TaARF genes

Gene ontology annotation and protein interaction network of TaARF genes. (A): Gene Ontology (GO) enrichment analysis for annotated TaARF genes. The GO terms are grouped into three main categories, green for biological processes, red for cellular components, and blue for molecular function; (B): TaARFA1 protein interaction network.

(A)

$\mathrm{GO}$ annotations analysis

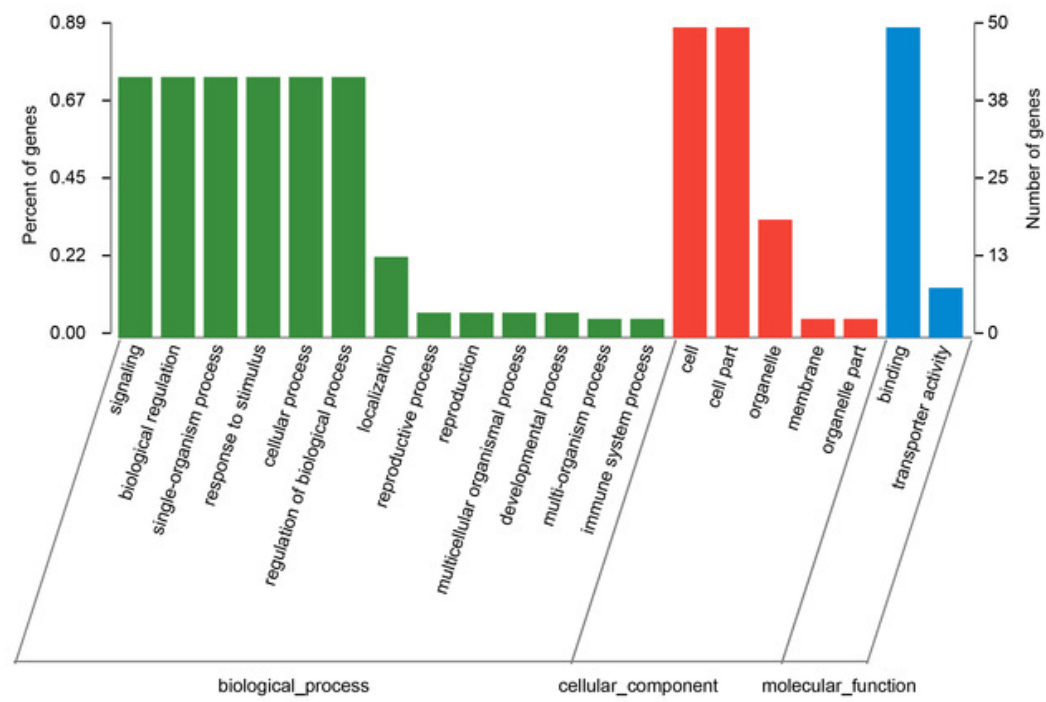

(B)

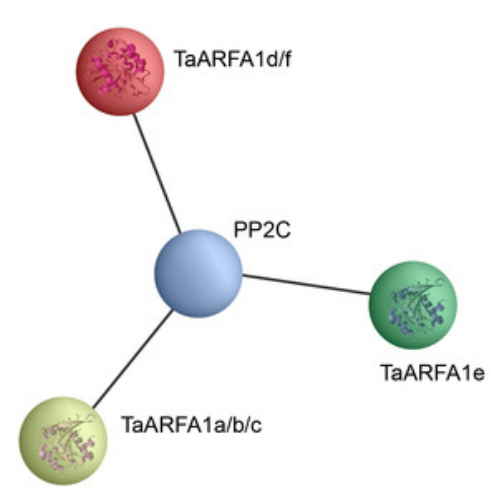

